\title{
SPECTRAL ACTION MODELS OF GRAVITY ON PACKED SWISS CHEESE COSMOLOGY
}

\author{
ADAM BALL AND MATILDE MARCOLLI
}

\begin{abstract}
We present a model of (modified) gravity on spacetimes with fractal structure based on packing of spheres, which are (Euclidean) variants of the Packed Swiss Cheese Cosmology models. As the action functional for gravity we consider the spectral action of noncommutative geometry, and we compute its asymptotic expansion on a space obtained as an Apollonian packing of 3-dimensional spheres inside a 4-dimensional ball. Using information from the zeta function of the Dirac operator of the spectral triple, we show that the leading terms in the asymptotic expansion of the spectral action consist of a zeta regularization of the divergent sum of the leading terms of the spectral actions of the individual spheres in the packing, which accounts for the contribution of the points 1 and 3 in the dimension spectrum (as in the case of a 3 -sphere). There is an additional term coming from the residue at the additional point in the dimension spectrum that corresponds to the packing constant. It detects the fractality of the residue set of the sphere packing. We show that the presence of fractality influences the shape of the slow-roll potential for inflation, obtained from the spectral action. We also discuss the effect of truncating the fractal structure at a certain scale related to the energy scale in the spectral action.
\end{abstract}

\section{INTRODUCTION}

1.1. Fractal structures in cosmology. The usual assumptions of isotropy and homogeneity of spacetime would require that the matter distribution scales uniformly in space. Large scale violations of homogeneity were discussed, for instance, in [33], while the idea of a fractal distribution of matter, scaling with a fractal dimension $D \neq 3$, was suggested in [32]. More recently, a growing literature based on the analysis of redshift catalogs at the level of galaxies, clusters, and superclusters has collected considerable evidence for the presence of fractality and multifractality in cosmology. We refer the reader to the survey [37] for a detailed discussion, see also [17]. While there is no complete agreement on the resulting dimensionality, partly due to difficulties in the interpretation of redshift data in estimating co-moving distances, multifractal models in cosmology have been widely studied in recent years. Cosmological models exhibiting a fractal structure can be constructed, adapting the original "swiss cheese model" of [33]. The resulting models are usually referred to as Packed Swiss Cheese Cosmology (PSCC), see [30 for a recent detailed survey. The main idea in the construction of swiss cheese models of cosmology is to have spacetimes that are locally inhomogenous but appear globally homogeneous and that satisfy everywhere the Einstein equation. In the original construction of PSCC models, in a region defined by a standard Friedmann-Robertson-Walker (FRW) cosmology, several non-overlapping spheres are inscribed, inside which the mass is contracted to a smaller higher density region, hence creating inhomogeneities. The solution inside the ball is patched to the external FRW solution along a surface with vanishing Weyl curvature tensor (which ensures isotropy is preserved). A swiss cheese type model based on the Tolman metric was developed in [34, [35]. In Packed Swiss Cheese Cosmology models, a configuration of such spheres is chosen so that they are 
tangent to each other and arranged into a higher dimensional version of the Apollonian packing of circles. In a variant of this model, see the discussion in $\S 8$ of [30], instead of compressing the matter inside each spherical region, at each stage of the construction process the matter is expanded to lie along the spherical shell, so that one ends up with a model of gravity interacting with matter, supported on the resulting fractal. The point of view we follow in this paper is similar to the latter: we consider spacetimes that are products of a time direction and a fractal arrangements of 3-spheres (or of other spherical space forms). We develop a model of gravity on such Packed Swiss Cheese Cosmology (PSCC) models using the spectral action as an action functional for gravity.

1.2. Spectral triples. In Noncommutative Geometry, the formalism of spectral triples extends ordinary Riemannian (and spin) geometry to noncommutative spaces, [13]. This approach encodes the metric structure in the data of a triple $S T=(\mathcal{A}, \mathcal{H}, D)$ of an involutive algebra $\mathcal{A}$ (associative, but not necessarily commutative), with a (faithful) representation $\pi: \mathcal{A} \rightarrow \mathcal{B}(\mathcal{H})$ by bounded operators on a Hilbert space $\mathcal{H}$, and with the additional structure of a Dirac operaror $D$, namely an unbounded, self-adjoint operator, densely defined on $\mathcal{H}$ with the properties:

(i): $\left(I+D^{2}\right)^{-1 / 2}$ is a compact operator

(ii): for all $a \in \mathcal{A}$, the commutators $[D, \pi(a)]$ are densely defined and extend to bounded operators on $\mathcal{H}$.

The metric dimension of a spectral triple is defined as

$$
\mathfrak{d}_{S T}:=\inf \left\{p>0 \mid \operatorname{tr}\left(\left(I+D^{2}\right)^{-p / 2}<\infty\right\} .\right.
$$

A spectral triple is said to be finitely summable if $\mathfrak{d}_{S T}<\infty$.

The notion of dimension for a spectral triple is more elaborate than just the metric dimension. Indeed, a more refined notion of dimension is given by the dimension spectrum, $\Sigma_{S T} \subset \mathbb{C}$. This is a set of complex numbers, defined as the set of poles of a family of zeta functions associated to the Dirac operator of the spectral triple. In the case where $\operatorname{Ker} D=0$, the zeta function of the Dirac operator is given by $\zeta_{D}(s)=\operatorname{Tr}\left(|D|^{-s}\right)$. Let $\delta(T)=[|D|, T]$ and let $\mathcal{B}$ denote the algebra generated by the $\delta^{m}(\pi(a))$ and $\delta^{m}([D, \pi(a)])$, for all $a \in \mathcal{A}$, and $m \in \mathbb{N}$. One considers additional zeta functions of the form $\zeta_{D, a}(s)=\operatorname{Tr}\left(a|D|^{-s}\right)$, for arbitrary $a \in \mathcal{A}$ and $\zeta_{D, b}(s)=\operatorname{Tr}\left(b|D|^{-s}\right)$, for arbitrary $a \in \mathcal{B}$. The dimension spectrum is the set of poles of the functions $\zeta_{D, a}(s)$ and $\zeta_{D, b}(s)$. It typically includes other points, in addition to the metric dimension, and may include real non-integer points as well as complex points off the real line. Spectral triples associated to fractals typically have non-integer and non-real points in their dimension spectrum. In the following, we will use the notation $\Sigma_{S T}^{+}:=\Sigma_{S T} \cap \mathbb{R}_{+}$for the part of the dimension spectrum contained in the non-negative real line. Geometrically, the dimension spectrum represents the set of dimensions in which the space manifests itself, when viewed as a noncommutative space. Even in the case of an ordinary manifold, the dimension spectrum contains additional points, besides the usual topological dimension. The non-negative dimension spectrum $\Sigma_{S T}^{+}$, in particular, describes the dimensions that contribute terms to the action functional for gravity, as we discuss more in detail in $\$ 1.3$ below.

A compact spin Riemannian manifold $M$ can be described by a spectral triple $S T_{M}=$ $\left(C^{\infty}(M), L^{2}(M, \mathbb{S}), \not D_{M}\right)$, by taking $\mathcal{A}=C^{\infty}(M)$, the algebra of smooth functions, $\mathcal{H}=$ $L^{2}(M, \mathbb{S})$ the Hilbert space of square-integrable spinors, and $D=\not D_{M}$ the Dirac operator, 
which is a self-adjoint square root of the (negative) Laplacian of the manifold. The metric dimension of $S T_{M}$ agrees with the dimension of $M$, by Weyl's law for the Dirac spectrum. One can also recover the geodesic distance on $M$ from $S T_{M}$ : for any two points $x, y \in M$

$$
d_{\text {geo }}(x, y)=\sup \{|f(x)-f(y)| \mid\|[D, \pi(f)]\| \leq 1\} .
$$

A reconstruction theorem [14 moreover shows that the manifold $M$ itself can be reconstructed from the data of a commutative spectral triple that satisfies a list of additional axioms describing properties of the geometry such as orientability, Poincaré duality, etc. The non-negative dimension spectrum $\Sigma_{S T_{M}}^{+}$consists of non-negative integers less than or equal to $\operatorname{dim}(M)$.

1.3. The spectral action as a model for (modified) gravity. The formalism of spectral triples plays a crucial role in the construction of models of gravity coupled to matter based on noncommutative geometry. The main ideas underlying the construction of these models can be summarized as follows:

- The spectral action is a natural action functional for gravity on any (commutative or noncommutative) space described by a finitely summable spectral triple.

- On an ordinary manifold, the asymptotic expansion of the spectral action recovers the usual Einstein-Hilbert action of gravity, with additional modified gravity terms (Weyl conformal gravity, Gauss-Bonnet gravity).

- In the case of an "almost commutative geometry" (locally a product $M \times F$ of an ordinary manifold $M$ and a finite noncommutative space) the model of gravity on $M \times F$ given by the spectral action describes gravity coupled to matter on $M$, with the matter content (fermions and bosons) completely determined by the geometry of the finite noncommutative space $F$.

We refer the reader to the detailed account of the construction of such models given in [9] and in Chapter 1 of [15]. For a finitely summable spectral triple, the spectral action functional [7] is defined as

$$
\mathcal{S}(\Lambda)=\operatorname{Tr}(f(D / \Lambda))=\sum_{\lambda \in \operatorname{Spec}(D)} \operatorname{Mult}(\lambda) f(\lambda / \Lambda)
$$

where $f$ is a non-negative even smooth approximation to a cutoff function and $\Lambda$ is a positive real number. As $\Lambda$ grows, more rescaled eigenvalues of the form $\lambda / \Lambda$ escape the cutoff of $f$ and the expression grows.

In the case of a finitely summable spectral triple with dimension spectrum consisting of simple poles, the spectral action can be expanded asymptotically for large $\Lambda$, [7]. The asymptotic expansion relies on the Mellin transform relation between the zeta function of the Dirac operator and the heat kernel. The asymptotic expansion of the spectral action is then of the form

$$
\operatorname{Tr}(f(D / \Lambda)) \sim \sum_{\beta \in \Sigma_{S T}^{+}} f_{\beta} \Lambda^{\beta} f|D|^{-\beta}+f(0) \zeta_{D}(0),
$$

where $f_{\beta}=\int_{0}^{\infty} f(v) v^{\beta-1} d v$ are the momenta of $f$, the summation is over the points of the non-negative dimension spectrum $\Sigma_{S T}^{+}$, and the coefficients are residues of the zeta function,

$$
f|D|^{-\beta}=\frac{1}{2} \operatorname{Res}_{s=\beta} \zeta_{D}(s)
$$


representing the noncommutative integration in dimension $\beta$.

In the case of a 4-dimensional manifold $M$, one can write the asymptotic expansion in the form 8

$$
\operatorname{Tr}(f(D / \Lambda)) \sim 2 \Lambda^{4} f_{4} a_{0}+2 \Lambda^{2} f_{2} a_{2}+f_{0} a_{4},
$$

where the $f_{i}$ are momenta of the cutoff function $f$, with $f_{0}=f(0)$ and $f_{k}=\int_{0}^{\infty} f(v) v^{k-1} d v$. Physically, the coefficients $a_{0}, a_{2}$ and $a_{4}$ correspond, respectively, to the cosmological term, the Einstein-Hilbert term, and the modified gravity terms (Weyl curvature and GaussBonnet) of the gravity action functional. In the case of an almost-commutative geometry, the asymptotic expansion of the spectral action delivers additional terms including YangMills terms for the gauge bosons, kinetic and interaction terms for the fermions and the Higgs bosons, boson-fermion interaction terms, and (non-minimal) coupling of matter to gravity (with the Higgs conformally coupled to gravity), see [9], [15]. We will see in the next section that, in the case of the Packed Swiss Cheese Cosmology, the spectral action has additional contributions that arise from an additional point in the dimension spectrum that reflects the fractality of the model.

\section{Spectral triples for Packed Swiss Cheese Cosmology}

2.1. Apollonian packings of $D$-dimensional spheres. Higher dimensional generalizations of the Apollonian packings of circles in the plane, consisting of "packings" of $(D-1)$ dimensional hyperspheres $S^{D-1}$ inside a $D$-dimensional space $\mathbb{R}^{D}$, were variously studied, for instance in [16], [19], [22], [26], [29], [36]. We recall here some useful facts, following [19].

A Descartes configuration in $D$ dimensions consists of $D+2$ mutually tangent $(D-1)$ dimensional (hyper)spheres. We write $S_{a}^{D-1}$ for a sphere of radius $a$. The curvature $c=1 / a$ is endowed with positive sign for the orientation of $S_{a}^{D-1}$ with an outward pointing normal vector and negative for the opposite orientation. The curvatures of the spheres in a Descartes configuration satisfy the quadratic Soddy-Gosset relation

$$
\left(\sum_{k=1}^{D+2} \frac{1}{a_{k}}\right)^{2}=D \sum_{k=1}^{D+2}\left(\frac{1}{a_{k}}\right)^{2} .
$$

This relation can be formulated in matrix terms as $c^{t} Q_{D} c=0$, with $c=\left(1 / a_{1}, \ldots, 1 / a_{D+2}\right)$ the vector of curvatures, and $Q_{n}$ the quadratic form determined by the matrix

$$
Q_{D}=I_{D+2}-D^{-1} 1_{D+2} 1_{D+2}^{t},
$$

where $1_{D+2}^{t}=(1,1, \ldots, 1)$ and $I_{D+2}$ is the identity matrix. The augmented curvature-center coordinates of a sphere $S_{a}^{D-1}$ with center $x=\left(x_{1}, \ldots, x_{D}\right)$ in $\mathbb{R}^{D}$ consist of a $(D+2)$-vector

$$
w=\left(\frac{\|x\|^{2}-a^{2}}{a}, \frac{1}{a}, \frac{1}{a} x_{1}, \ldots, \frac{1}{a} x_{D}\right),
$$

where the first coordinate describes the curvature of the sphere obtained from the given one by inversion in the unit sphere. The reason for the first coordinate is so that one can extend unambiguously the augmented curvature-center coordinates to include the special case of degenerate spheres with zero curvature (hyperplanes). Given a Descartes configuration of spheres, one assigns to it a $(D+2) \times(D+2)$ matrix $\mathcal{W}$ whose $j$-th row is the vector of augmented curvature-center coordinates of the $j$-th sphere in the configuration. The space 
$\mathcal{M}_{D}$ of all possible Descartes configuration in $D$ dimensions is then identified with the space of all solutions $\mathcal{W}$ to the equation

$$
\mathcal{W}^{t} Q_{D} \mathcal{W}=\left(\begin{array}{ccc}
0 & -4 & 0 \\
-4 & 0 & 0 \\
0 & 0 & 2 I_{D}
\end{array}\right)
$$

The space of solutions $\mathcal{M}_{D}$ is endowed with a left and a right action of the Lorentz group $O(D+1,1)$.

It is shown in [19] that in dimension $D \geq 4$ the analog of the Apollonian group for circle packings is no longer a discrete subgroup of $\operatorname{GL}(D+2, \mathbb{R})$ and its orbits on $\mathcal{M}_{D}$ no longer correspond to sphere packings. However, the dual Apollonian group is a discrete subgroup of $\operatorname{GL}(D+2, \mathbb{R})$, and the Apollonian packings of $(D-1)$-dimensional spheres we will be considering here are obtained, as in Theorem 4.3 of [19, as orbits of the dual Apollonian group on $\mathcal{M}_{D}$. The dual Apollonian group $\mathcal{G}_{D}^{\perp}$ is generated by reflections $S_{j}^{\perp}$ of the form

$$
S_{j}^{\perp}=I_{D+2}+21_{D+2} e_{j}^{t}-4 e_{j} e_{j}^{t},
$$

with $e_{j}$ the $j$-th unit coordinate vector. The matrix $S_{j}^{\perp}$ implements inversion with respect to the $j$-th sphere. The Apollonian packing is obtained by iteratively adding new Descartes configurations of spheres obtained from an initial one by iteratively applying inversions with respect to some of the spheres. When $D \neq 3$ the only relations in the dual Apollonian group $\mathcal{G}_{D}^{\perp}$ are $\left(S_{j}^{\perp}\right)^{2}=1$. Thus, the spheres added at the $n$-th iterative step of the construction of the Apollonian packing are in correspondence with all the possible reduces sequences

$$
S_{j_{1}}^{\perp} S_{j_{2}}^{\perp} \cdots S_{j_{n}}^{\perp}, \quad j_{k} \neq j_{k+1}, \forall k,
$$

acting on the point $\mathcal{W} \in \mathcal{M}_{D}$ that corresponds to the initial Descartes configuration. Clearly, there are $(D+2)(D+1)^{n-1}$ such sequences, hence the number of spheres in the $n$-th level of the iterative construction is

$$
N_{n}:=\#\left\{S_{a_{n, k}}^{D-1}: \text { fixed } n\right\}=(D+2)(D+1)^{n-1} .
$$

In the following, we will focus on the case $D=4$, of Apollonian packings of 3-spheres.

2.2. Lengths, packing constant, and zeta function. We proceed as in [10, [11] to associate a spectral triple to an Apollonian packing $\mathcal{P}_{D}$ of $(D-1)$-spheres in a $D$-dimensional space. As above, let

$$
\mathcal{L}_{D}\left(\mathcal{P}_{D}\right)=\left\{a_{n, k}, n \in \mathbb{N}, 1 \leq k \leq(D+2)(D+1)^{n-1}\right\}
$$

be the list (with multiplicities) of the radii $a_{n, k}$ of the $(D+2)(D+1)^{n-1}$ spheres $S_{a_{n, k}}^{D-1}$ that are added in the $n$-th stage of the iterative construction of the packing.

The packing constant (or exponent of the packing), $\sigma_{D}\left(\mathcal{P}_{D}\right)$ of a packing $\mathcal{P}_{D}$ of $(D-1)$ spheres is defined as the exponent of convergence of the series

$$
\sum_{n \in \mathbb{N}} \sum_{k=1}^{(D+2)(D+1)^{n-1}} a_{n, k}^{s}
$$


that is,

$$
\begin{aligned}
\sigma_{D}\left(\mathcal{P}_{D}\right) & =\sup \left\{s \in \mathbb{R}_{+}^{*}: \sum_{n \in \mathbb{N}} \sum_{\substack{k=1 \\
(D+2)(D+1)^{n-1}}}^{(D+2)(D+1)^{n-1}} a_{n, k}^{s}=\infty\right\} \\
& =\inf \left\{s \in \mathbb{R}_{+}^{*}: \sum_{n \in \mathbb{N}} \sum_{k=1}^{s}<\infty\right\} .
\end{aligned}
$$

For $s>\sigma_{D}\left(\mathcal{P}_{D}\right)$, one defines the zeta function $\zeta_{\mathcal{L}_{D}}(s)$ as the sum of the series

$$
\zeta_{\mathcal{L}_{D}}(s)=\sum_{n \in \mathbb{N}} \sum_{k=1}^{(D+2)(D+1)^{n-1}} a_{n, k}^{s} .
$$

The zeta functions $\zeta_{\mathcal{L}_{D}}(s)$, like the more general zeta functions of fractal strings considered in [23], need not in general have analytic continuation to meromorphic function on the whole complex plane, but there are a screen $\mathcal{S}$, namely a curve of the form $S(t)+i t$, with $S: \mathbb{R} \rightarrow\left(-\infty, \sigma_{D}\left(\mathcal{P}_{D}\right)\right]$, and a window $\mathcal{W}$ consisting of the region to the right of the screen curve $\mathcal{S}$ in the complex plane, where $\zeta_{\mathcal{L}_{D}}(s)$ has analytic continuation. We refer the reader to [23] for a more detailed account of screens and windows for zeta functions of fractal strings.

2.3. Packing constant and Hausdorff dimension. The residual set of an Apollonian circle packing consists of the complement of the union of all the open balls consisting of the interiors of the circles in the packing. It was shown in [4] that the packing constant $\sigma_{2}$, defined as in (2.5) is equal to the Hausdorff dimension of the residual set of the circle packing. In the higher dimensional setting the problem of estimating the Hausdorff dimension of the residual set of a packing of $(D-1)$-dimensional spheres is much more involved, but there are some general estimates, obtained in [24] and [20].

Consider the infimum of the packing constants over all packings $\mathcal{P}_{D}$,

$$
\sigma_{D}=\inf _{\mathcal{P}_{D}} \sigma_{D}\left(\mathcal{P}_{D}\right)
$$

Assuming all the spheres $S_{a_{n, k}}^{D-1}$ in the packing are contained in the unit ball $B^{D}$, and denoting by $B_{a_{n, k}}^{D}$ the $D$-dimensional ball with $\partial B_{a_{n, k}}^{D}=S_{a_{n, k}}^{D-1}$, the residual set of the packing is given by

$$
\mathcal{R}\left(\mathcal{P}_{D}\right)=B^{D} \backslash \cup_{n, k} B_{a_{n, k}}^{D}
$$

Let $\operatorname{dim}_{H}\left(\mathcal{R}\left(\mathcal{P}_{D}\right)\right)$ denote the Hausdorff dimension of the residual set and

$$
\delta_{D}=\inf _{\mathcal{P}_{D}} \operatorname{dim}_{H}\left(\mathcal{R}\left(\mathcal{P}_{D}\right)\right)
$$

the infimum over all packings of the Hausdorff dimensions. The upper entropy dimension $h^{+}\left(\mathcal{R}\left(\mathcal{P}_{D}\right)\right)$ of the residual set $\mathcal{R}\left(\mathcal{P}_{D}\right)$ is defined as

$$
h^{+}\left(\mathcal{R}\left(\mathcal{P}_{D}\right)\right)=\limsup _{\epsilon \rightarrow 0}-\frac{\log N_{\epsilon}\left(\mathcal{R}\left(\mathcal{P}_{D}\right)\right)}{\log \epsilon},
$$

where for a set $X$, the number $N_{\epsilon}(X)$ counts the smallest number of sets of diameter less than $2 \epsilon$ that cover $X$. The lower entropy dimension is defined similarly, with a liminf instead of limsup. It is known that the entropy dimension provides an upper bound for the Hausdorff dimension. Then we have the following estimates ([24] and [20]). 
Proposition 2.1. The radii $a_{n, k}$ of a packing $\mathcal{P}_{D}$ satisfy $\sum_{n, k} a_{n, k}^{D}=1$ and $\sum_{n, k} a_{n, k}^{D-1}=\infty$, hence $D-1<\sigma_{D}\left(\mathcal{P}_{D}\right) \leq D$. The infima satisy $\delta_{D} \leq \sigma_{D}$, and for individual packings $\operatorname{dim}_{H}\left(\mathcal{R}\left(\mathcal{P}_{D}\right)\right) \leq h^{+}\left(\mathcal{R}\left(\mathcal{P}_{D}\right)\right)=\sigma_{D}\left(\mathcal{P}_{D}\right)$.

The identity $\sum_{n, k} a_{n, k}^{D}=1$ follows from the packing property, namely the requirement that the residual set $\mathcal{R}\left(\mathcal{P}_{D}\right)$ in the $D$-dimensional unit ball has zero $D$-dimensional volume. The value $\operatorname{dim}_{H}\left(\mathcal{P}_{D}\right)$ is not known exactly. Some estimates are obtained, with various methods, in [16], [29], [36]. We provide a simple rough estimate in \$2.4 below, for the specific case of 3 -spheres.

2.4. Dimension estimate. Let $\mathcal{P}=\mathcal{P}_{4}$ be an Apollonian packing of 3-dimensional spheres $S_{a_{n, k}}^{3}$. We compute here a rough approximation to the packing constant $\sigma_{4}(\mathcal{P})$ of the Apollonian packing, defined as in (2.5).

Proposition 2.2. By replacing the collection of radii $\left\{a_{n, k}\right\}$ in the $n$-th level of the Apollonian packing $\mathcal{P}$ of 3 -spheres with a single value $a_{n}=N_{n} / \gamma_{n}$, where $\gamma_{n} / N_{n}$ is the average curvature in the $n$-th level, one obtains an approximate estimate of the packing constant,

$$
\sigma_{4, a v}(\mathcal{P}) \sim 3.85193 \ldots
$$

Proof. As discussed above, the number of 3 -spheres in the $n$-th level of the packing $\mathcal{P}$ is given by the number of reduced sequences in the generators of the group $\mathcal{G}_{n}$, namely

$$
N_{n}:=\#\left\{S_{a_{n, k}}^{3}: \text { fixed } n\right\}=\left.(D+2)(D+1)^{n-1}\right|_{D=4}=6 \cdot 5^{n-1} .
$$

Let $\gamma_{n}$ denote the sum of the curvatures of the spheres in the $n$-th level,

$$
\gamma_{n}=\sum_{k=1}^{6 \cdot 5^{n-1}} \frac{1}{a_{n, k}}
$$

As shown in Theorem 2 of [26], the generating function of the $\gamma_{n}=\gamma_{n}(s)$ is

$$
G_{D=4}(u)=\frac{(1-x)(1-4 x) u}{1-\frac{22}{3} x-5 x^{2}},
$$

where $u=\gamma_{0}$ is the sum of the curvatures of the $D+2=6$ spheres in a Descartes configuration that gives the level-zero seed of the recursive construction. We obtain from this an estimate of the metric dimension by replacing the curvatures $1 / a_{n, k}$ with their averages over levels. We denote the resulting approximation to the dimension by $\sigma_{4, a v}(\mathcal{P})$. This is given by

$$
\sigma_{4, a v}(\mathcal{P})=\lim _{n \rightarrow \infty} \frac{\log \left(6 \cdot 5^{n-1}\right)}{\log \left(\frac{\gamma_{n}}{6 \cdot 5^{n-1}}\right)} .
$$

We expand (2.9) in a power series. Since the specific value of $u$ does not influence the large $n$ behavior in the limit above, we look at the values for $u=1$, and we obtain

$$
\begin{gathered}
G_{D=4}=\sum_{n=1}^{\infty} \gamma_{n}(1) x^{n}, \\
\gamma_{n}(1)=\frac{(11+\sqrt{166})^{n}(-64+9 \sqrt{166})+(11-\sqrt{166})^{n}(64+9 \sqrt{166})}{3^{n} \cdot 10 \cdot \sqrt{166}} .
\end{gathered}
$$

This then gives $\sigma_{4, a v}(\mathcal{P}) \sim 3.85193 \ldots$ as stated. 
2.5. A spectral triple on the Cayley graph of the dual Apollonian group. Let $\mathcal{T}_{D}$ denote the Cayley graph of the dual Apollonian group $\mathcal{G}_{D}^{\perp}$. Since for $D \neq 3$ the group $\mathcal{G}_{D}^{\perp}$ is generated by the $D+2$ reflections $S_{j}^{\perp}$ of (2.3), with the only relations of the form $\left(S_{j}^{\perp}\right)^{2}=1$, the Cayley graph $\mathcal{T}_{D}$ is an infinite tree with all vertices of valence $D+2$. We endow the tree $\mathcal{T}_{D}$ with the structure of a finitely summable tree, in the sense of $\S 7$ of [10], by choosing a base vertex $v_{0}$ and endowing all the $N_{n}=(D+2)(D+1)^{n-1}$ edges at a distance of $n$ steps from $v_{0}$ with lengths $\ell\left(e_{n, k}\right)=a_{n, k}$, equal to the radii of the spheres in the $n$-th level of the sphere packing. Then, as in Theorem 7.10 of [10] one obtains a finitely summable spectral triple

$$
S T_{\mathcal{T}_{D}}=\left(\mathcal{A}_{\mathcal{T}_{D}}, \mathcal{H}_{\mathcal{T}_{D}}, \mathcal{D}_{\mathcal{T}_{D}}\right)=\oplus_{e \in E\left(\mathcal{T}_{D}\right)}\left(\mathcal{A}_{\mathcal{T}_{D}}, \mathcal{H}_{\ell(e)}, D_{\ell(e)}+\frac{\pi}{2 \ell(e)} I\right)
$$

The involutive subalgebra $\mathcal{A}_{\mathcal{T}_{D}}$ of the $C^{*}$-algebra $C\left(\mathcal{T}_{D}\right)$ is determined, as in [10], by the condition that $f \in \mathcal{A}_{\mathcal{T}_{D}}$ has $\left[\mathcal{D}_{\mathcal{T}_{D}}, \pi(f)\right]$ densely defined and bounded, where $\pi: C\left(\mathcal{T}_{D}\right) \rightarrow$ $\mathcal{B}\left(\mathcal{H}_{\mathcal{T}_{D}}\right)$ is the representation by bounded operators on the Hilbert space of the triple. The pairs $\left(\mathcal{H}_{\ell(e)}, D_{\ell(e)}\right)$ are constructed as in the "interval spectral triple" of $\S 3$ of [10], with $\mathcal{H}_{\alpha}=L^{2}([-\alpha, \alpha], \mu)$ with the normalized Lebesgue measure $\mu$ and $D_{\alpha}$ with eigenvectors the basis elements $e_{m}=\exp (i \pi m x / \alpha)$ with eigenvalue $\pi m / \alpha$. The Dirac operator $\mathcal{D}_{\mathcal{T}_{D}}$ then has spectrum

$$
\begin{gathered}
\operatorname{Spec}\left(\mathcal{D}_{\mathcal{T}_{D}}\right)=\left\{\frac{\pi(2 m+1)}{2 \ell(e)}: e \in E\left(\mathcal{T}_{D}\right), m \in \mathbb{Z}_{+}\right\} \\
=\left\{\frac{\pi(2 m+1)}{2 a_{n, k}}: n \in \mathbb{N}, 1 \leq k \leq(D+2)(D+1)^{n-1}, m \in \mathbb{Z}_{+}\right\} .
\end{gathered}
$$

The shift $\pi / 2 \ell(e) I$ to the Dirac operator $D_{\ell(e)}$ is introduced in [10] to avoid a kernel, so that the zeta function $\zeta_{D_{\mathcal{T}_{D}}}(s)=\operatorname{Tr}\left(\left|\mathcal{D}_{\mathcal{T}_{D}}\right|^{-s}\right)$ is well defined. The exponent of summability of the spectral triple (the metric dimension) is equal to the packing constant of (2.5),

$$
\mathfrak{d}_{S T_{\mathcal{T}_{D}}}=\sigma_{D}
$$

The zeta function of the Dirac operator of the spectral triple $S T_{\mathcal{T}_{D}}$ is given by

$$
\operatorname{Tr}\left(\left|\mathcal{D}_{\mathcal{T}_{D}}\right|^{-s}\right)=\frac{2^{s+1}}{\pi^{s}}\left(1-2^{-s}\right) \zeta(s) \zeta_{\mathcal{L}_{D}}(s)
$$

where $\zeta(s)$ is the Riemann zeta function, see $\S 7.1$ of [10].

2.6. The spectral triple of a sphere packing. Suppose given an Apollonian packing $\mathcal{P}_{D}$ of $(D-1)$-dimensional spheres $S_{a_{n, k}}^{D-1}$ in $\mathbb{R}^{D}$. We modify the construction above, by introducing the contribution of the $(D-1)$-spheres $S_{a_{n, k}}^{D-1}$ of the packing, through their respective spectral triples. We replace the data $\left(\mathcal{H}_{\ell\left(e_{n, k}\right)}, D_{\ell\left(e_{n, k}\right)}\right)$ of the construction above, for an edge $e_{n, k}$ of length $\ell\left(e_{n, k}\right)=a_{n, k}$, with new data of the form $\left(\mathcal{H}_{S_{a_{n, k}}^{D-1}}, \mathcal{D}_{S_{a_{n, k}}^{D-1}}\right)$, where $\mathcal{H}_{S_{a_{n, k}}^{D-1}}=L^{2}\left(S_{a_{n, k}}^{D-1}, \mathbb{S}\right)$ is the Hilbert space of square integrable spinors on the $(D-1)$-sphere $S_{a_{n, k}}^{D-1}$, and $\mathcal{D}_{S_{a_{n, k}}^{D-1}}$ is the Dirac operator, with spectrum

$$
\operatorname{Spec}\left(D_{S_{a_{n, k}}^{D-1}}\right)=\left\{\lambda_{\ell, \pm}= \pm a_{n, k}^{-1}\left(\frac{D-1}{2}+\ell\right): \ell \in \mathbb{Z}_{+}\right\}
$$

and multiplicities

$$
\operatorname{Mult}\left(\lambda_{\ell, \pm}\right)=2^{\left[\frac{D-1}{2}\right]}\left(\begin{array}{c}
\ell+D \\
\ell
\end{array}\right)
$$


Definition 2.3. The spectral triple of the Apollonian packing

$$
\mathcal{P}_{D}=\left\{S_{a_{n, k}}^{D-1}: n \in \mathbb{N}, 1 \leq k \leq(D+2)(D+1)^{n-1}\right\}
$$

is given by

$$
\left(\mathcal{A}_{\mathcal{P}_{D}}, \mathcal{H}_{\mathcal{P}_{D}}, \mathcal{D}_{\mathcal{P}_{D}}\right)=\oplus_{e \in \mathcal{E}\left(\mathcal{T}_{D}\right)}\left(\mathcal{A}_{\mathcal{P}_{D}}, \mathcal{H}_{S_{\ell(e)}^{D-1}}, \mathcal{D}_{S_{\ell(e)}^{D-1}}\right)
$$

where $\mathcal{T}_{D}$ the the Cayley graph of $\mathcal{G}_{D}^{\perp}$, as above, with edge lengths $\ell\left(e_{n, k}\right)=a_{n, k}$, and the data $\left(\mathcal{H}_{S_{a_{n}, k}^{D-1}}, \mathcal{D}_{S_{a_{n, k}}^{D-1}}\right)$ are defined as above. The involutive subalgebra $\mathcal{A}_{\mathcal{P}_{D}}$ consists of $f \in C\left(\mathcal{P}_{D}\right)$ with $\left[\mathcal{D}_{\mathcal{P}_{D}}, \pi(f)\right]$ densely defined and bounded.

The fact that this is indeed a spectral triple follows from the general results of [10] and [11]. In particular, the spectral action of the Swiss Cheese Cosmology model is obtained by considering the case of a packing of 3-dimensional spheres,

$$
\mathrm{ST}_{P S C}:=\left(\mathcal{A}_{\mathcal{P}_{4}}, \mathcal{H}_{\mathcal{P}_{4}}, \mathcal{D}_{\mathcal{P}_{4}}\right) \text {. }
$$

In order to compute the spectral action for the spectral triple of a packing of 3-spheres, we first recall some facts about the spectral action of a single 3 -sphere.

2.7. The spectral action on the 3-sphere. We start by recalling some very simple and well known facts about the round sphere $S^{3}$ and its spectral action functional. We will need these in the rest of this section as building blocks to construct the spectral triple and the spectral action for the Packed Swiss Cheese Cosmology.

The Dirac operator on the 3 -sphere $S^{3}$ with the round metric of unit radius has spectrum $\operatorname{Spec}\left(D_{S^{3}}\right)=\left\{n+\frac{1}{2}\right\}$ with spectral multiplicities $\operatorname{Mult}\left(n+\frac{1}{2}\right)=n(n+1)$, hence the spectral action takes the form

$$
\mathcal{S}_{S^{3}}(\Lambda)=\operatorname{Tr}\left(f\left(D_{S^{3}} / \Lambda\right)\right)=\sum_{n \in \mathbb{Z}} n(n+1) f\left(\left(n+\frac{1}{2}\right) / \Lambda\right) .
$$

Lemma 2.4. The zeta function of the Dirac operator is of the form

$$
\zeta_{D_{S^{3}}}(s)=2 \zeta\left(s-2, \frac{3}{2}\right)-\frac{1}{2} \zeta\left(s, \frac{3}{2}\right),
$$

where $\zeta(s, q)$ is the Hurwitz zeta function. The spectral triple $S T_{S^{3}}$ has simple dimension spectrum, with $\Sigma_{S T_{S}}^{+}=\{1,3\}$. The asymptotic expansion of the spectral action is correspondingly of the form

$$
\mathcal{S}_{S^{3}}(\Lambda) \sim \Lambda^{3} f_{3}-\frac{1}{4} \Lambda f_{1}
$$

Proof. The result immediately follows by writing

$$
\operatorname{Tr}\left(\left|D_{S^{3}}\right|^{-s}\right)=\sum_{k \geq 0} 2(k+1)(k+2)\left(k+\frac{3}{2}\right)^{-s}=\sum_{k \geq 0} 2\left(k+\frac{3}{2}\right)^{-(s-2)}-\frac{1}{2} \sum_{k \geq 0}\left(k+\frac{3}{2}\right)^{-s} .
$$

The Hurwitz zeta function $\zeta(s, q)$ has a simple pole at $s=1$ with residue one, hence $\zeta_{D_{S} 3}(s)$ has simple poles at $s=1$ and $s=3$, respectively with residues $\operatorname{Res}_{s=1} \zeta_{D_{S^{3}}}(s)=-1 / 2$ and $\operatorname{Res}_{s=3} \zeta_{D_{S^{3}}}(s)=2$. Then applying (1.2), one obtains the spectral action expansion. In the constant term we have $\zeta_{D_{S} 3}(0)=2 \zeta(-2,3 / 2)-\zeta(0,3 / 2) / 2=0$, since $\zeta(-2,3 / 2)=-1 / 4$ and $\zeta(0,3 / 2)=-1$. 
Corollary 2.5. In the case of a 3-sphere $S_{a}^{3}$ with the round metric of radius a $>0$, the zeta function is of the form

$$
\zeta_{D_{S_{a}^{3}}}(s)=a^{s}\left(2 \zeta\left(s-2, \frac{3}{2}\right)-\frac{1}{2} \zeta\left(s, \frac{3}{2}\right)\right),
$$

and the asymptotic expansion of the spectral action is given by

$$
\mathcal{S}_{S_{a}^{3}}(\Lambda) \sim(\Lambda a)^{3} f_{3}-\frac{1}{4}(\Lambda a) f_{1} .
$$

Proof. The spectrum of the Dirac operator $D_{S_{a}^{3}}$ is a scaled copy $\frac{1}{a}\left(\frac{1}{2}+\mathbb{Z}\right)$ of the spectrum of $D_{S_{1}^{3}}$, and the multiplicities coincide. Thus, we have

$$
\begin{aligned}
\operatorname{Tr}\left(\left|D_{S_{a}^{3}}\right|^{-s}\right) & =\sum_{n=1}^{\infty} 2 n(n+1)\left(\frac{n+1 / 2}{a}\right)^{-s}=2 a^{s} \sum_{n=1}^{\infty} n(n+1)(n+1 / 2)^{-s} \\
= & 2 a^{s} \sum_{n=1}^{\infty}(n+1 / 2)^{2}(n+1 / 2)^{-s}-\frac{a^{s}}{2} \sum_{n=1}^{\infty}(n+1 / 2)^{-s} \\
= & 2 a^{s} \sum_{n=0}^{\infty}(n+3 / 2)^{-(s-2)}-\frac{a^{s}}{2} \sum_{n=0}^{\infty}(n+3 / 2)^{-s}
\end{aligned}
$$

When $\Re(s)>3$ (the metric dimension of the 3 -sphere), this simplifies to (2.15).

A method for non-perturbative computations of the spectral action functional based on the Poisson summation formula was developed in [8], for sufficiently regular geometries for which the Dirac spectrum and the spectral multiplicities are explicitly known. In particular, the spectral action for the round sphere $S^{3}$ was computed in 8 using this method. The computation was generalized to spherical space forms, [38], and to 3-dimensional tori and Bieberbach manifolds in [27], [28], [31]. The computation of [8] for the 3-sphere can be summarized quickly as follows. Let $f$ be a rapidly decaying even function. The eigenvalues of $D_{S^{3}}$ form an arithmetic progression, and there is a polynomial $P(u)=u^{2}-\frac{1}{4}$ that interpolates the spectral multiplicities, $\operatorname{Mult}(\lambda)=P(\lambda)$. Thus, one can write the spectral action as

$$
\mathcal{S}_{S^{3}}(\Lambda)=\sum_{n \in \mathbb{Z}} g\left(n+\frac{1}{2}\right),
$$

where $g(u)=\left(u^{2}-\frac{1}{4}\right) f(u / \Lambda)$ is also a rapidly decaying function. This is then the sum of values of a rapidly decaying function on points of a lattice, which can be evaluated using the Poisson summation formula

$$
\sum_{n \in \mathbb{Z}} g\left(n+\frac{1}{2}\right)=\sum_{n \in \mathbb{Z}}(-1)^{n} \hat{g}(n),
$$

where

$$
\hat{g}(x)=\int_{\mathbb{R}} g(u) e^{-2 \pi i x u} d u=\int_{\mathbb{R}}\left(u^{2}-\frac{1}{4}\right) f(u / \Lambda) e^{-2 \pi i x u} d u
$$

is the Fourier transform

$$
\hat{g}(x)=\Lambda^{3} \int_{\mathbb{R}} v^{2} f(v) e^{-2 \pi i \Lambda x v} d v-\frac{1}{4} \Lambda \int_{\mathbb{R}} f(v) e^{-2 \pi i \Lambda x v} d v,
$$


after substituting $u=\Lambda v$. Let $\hat{f}^{(2)}$ denote the Fourier transform of $v^{2} f(v)$, in the first term above. It is shown in [8] that the sum on the Fourier transformed side can be very accurately approximated by the term with $n=0$, yielding for any $k$

$$
\operatorname{Tr}(f(D / \Lambda))=\Lambda^{3} \int_{\mathbb{R}} v^{2} f(v) d v-\frac{1}{4} \Lambda \int_{\mathbb{R}} f(v) d v+O\left(\Lambda^{-k}\right) .
$$

In the case of the round 3 -sphere $S_{a}^{3}$ of radius $a$, we have

$$
\operatorname{Tr}\left(f\left(\frac{D_{S_{a}^{3}}}{\Lambda}\right)\right)=\operatorname{Tr}\left(f\left(\frac{D_{S_{1}^{3}}}{\Lambda a}\right)\right),
$$

and the approximation formula above extends to $S_{a}^{3}$, replacing $\Lambda$ with $\Lambda a$, so one obtains

$$
\operatorname{Tr}\left(f\left(D_{S_{a}^{3}} / \Lambda\right)\right)=(\Lambda a)^{3} \int_{\mathbb{R}} v^{2} f(v) d v-\frac{1}{4}(\Lambda a) \int_{\mathbb{R}} f(v) d v+O\left((\Lambda a)^{-K}\right),
$$

for arbitrary $K \in \mathbb{N}$, which agrees with the expression (2.16), with the error term as in [8].

2.8. Zeta function and spectral action of a 3-sphere packing. We focus here on the case of a packing $\mathcal{P}=\mathcal{P}_{4}$ of 3 -spheres, where at the $n$-th iterative step in the construction one has $6 \cdot 5^{n-1}$ spheres, with radii $a_{n, k}$ with $k=1, \ldots, 6 \cdot 5^{n-1}$, starting with an initial Descartes configuration of 6 mutually tangent 3-spheres. As above, let $\mathcal{L}=\mathcal{L}_{4}=\left\{a_{n, k} \mid n \in \mathbb{N}, k \in\right.$ $\left.\left\{1, \ldots, 6 \cdot 5^{n-1}\right\}\right\}$ be the length spectrum of the radii of all the 3 -spheres in the packing. We consider the associated zeta function (2.6) for $D=4$, which we denote simply by $\zeta_{\mathcal{L}}(s)$,

$$
\zeta_{\mathcal{L}}(s):=\sum_{n \in \mathbb{N}} \sum_{k=1}^{6 \cdot 5^{n-1}} a_{n, k}^{s}
$$

Proposition 2.6. Let $\sigma_{4}(\mathcal{P})$ be the packing constant of an Apollonian packing $\mathcal{P}$ of 3dimensional spheres, as in (2.5). For $s>\sigma_{4}(\mathcal{P})$, the zeta function of the Dirac operator $\mathcal{D}_{\mathcal{P}}$ of the spectral triple $\mathrm{ST}_{P S C}$ of (2.11) is given by

$$
\operatorname{Tr}\left(\left|\mathcal{D}_{\mathcal{P}}\right|^{-s}\right)=\left(2 \zeta\left(s-2, \frac{3}{2}\right)-\frac{1}{2} \zeta\left(s, \frac{3}{2}\right)\right) \zeta_{\mathcal{L}}(s),
$$

where $\zeta(s, q)$ is the Hurwitz zeta function and $\zeta_{\mathcal{L}}(s)$ is as in (2.18).

Proof. Since $0 \notin \operatorname{Spec}\left(D_{S_{a}^{3}}\right), D_{S_{a}^{3}}$ is invertible and so is then the Dirac operator $\mathcal{D}_{\mathcal{P}}$ for the spectral triple $\mathrm{ST}_{P S C}$. The metric dimension is then given by $\inf \left\{\beta>0 \mid \operatorname{Tr}\left(\left|\mathcal{D}_{\mathcal{P}}\right|^{-\beta}\right)<\infty\right\}$, where the zeta function is given by

$$
\operatorname{Tr}\left(\left|\mathcal{D}_{\mathcal{P}}\right|^{-s}\right)=\sum_{n=1}^{\infty} \sum_{k=1}^{6 \cdot 5^{n-1}} \operatorname{Tr}\left(\left|D_{S_{a_{n}, k}^{3}}\right|^{-s}\right) .
$$

Each term in this sum can be computed as in (2.15). We can then evaluate the zeta function of the spectral triple $S T_{P S C}$, using the fact that the contribution of each sphere $S_{a_{n, k}}^{3}$ is of the form $\operatorname{Tr}\left(\left|D_{S_{a_{n, k}}^{3}}\right|^{-s}\right)=a_{n, k}^{s}\left(2 \zeta\left(s-2, \frac{3}{2}\right)-\frac{1}{2} \zeta\left(s, \frac{3}{2}\right)\right)$, and we obtain

$$
\operatorname{Tr}\left(|D|^{-s}\right)=\sum_{k=0}^{\infty} \operatorname{Tr}\left(\left|D_{S_{a_{n, k}}^{3}}\right|^{-s}\right)=\left(2 \zeta\left(s-2, \frac{3}{2}\right)-\frac{1}{2} \zeta\left(s, \frac{3}{2}\right)\right) \sum_{k=0}^{\infty} a_{n, k}^{s},
$$


for $s>\sigma$, with $\sigma=\max \left\{3,1, \sigma_{4}(\mathcal{P})\right\}=\sigma_{4}(\mathcal{P})$, where $\sigma_{D}\left(\mathcal{P}_{D}\right)$, as in (2.5), is the packing constant of $\mathcal{P}_{D}$, the exponent of convergence of the series $\sum a_{n, k}^{s}$. We know from \$2.3 that $3 \leq \sigma_{4} \leq 4$, hence $\max \left\{3,1, \sigma_{4}(\mathcal{P})\right\}=\sigma_{4}(\mathcal{P})$.

Definition 2.7. A packing $\mathcal{P}$ of 3 -dimensional spheres is analytic if it satisfies the following properties:

(1) The zeta function $\zeta_{\mathcal{L}}(s)$ of the packing $\mathcal{P}$ has analytic continuation to meromorphic function on a region of the complex plane that contains the non-negative real axis.

(2) The analytic continuation $\zeta_{\mathcal{L}}(s)$ has only one pole on the non-negative real axis, located at $s=\sigma_{4}(\mathcal{P})$.

(3) The pole of $\zeta_{\mathcal{L}}(s)$ at $s=\sigma_{4}(\mathcal{P})$ is simple.

In terms of screens and windows, as in [23], the first condition above consists of the property that the screen function $S: \mathbb{R} \rightarrow\left(-\infty, \sigma_{4}(\mathcal{P})\right.$ ] satisfies $S(0)<0$.

For the rest of this section we make the assumptions that the packing $\mathcal{P}$ of 3 -dimensional spheres we are considering satisfies the three conditions listed above.

We can then directly obtain the non-negative dimension spectrum $\Sigma_{S T_{P S C}}^{+}$.

Proposition 2.8. For a packing $\mathcal{P}$ of 3-spheres satisfying the three properties listed above, the non-negative dimension spectrum of the spectral triple of the Packed Swiss Cheese Cosmology consists of the points

$$
\Sigma_{S T_{P S C}}^{+}=\left\{1,3, \sigma_{4}(\mathcal{P})\right\}
$$

The zeta function $\zeta_{\mathcal{D}_{\mathcal{P}}}(s)$ has simple poles at the points of $\Sigma^{+}$. The metric dimension of the spectral triple is $\mathfrak{d}_{P S C}=\sigma_{4}(\mathcal{P})$.

Proof. Under the assumptions that the zeta function $\zeta_{\mathcal{L}}(s)$ has analytic continuation to a window including the positive real axis, we see that the zeta function $\zeta_{\mathcal{D}_{\mathcal{P}}}(s)=\operatorname{Tr}\left(\left|\mathcal{D}_{\mathcal{P}}\right|^{-s}\right)$ of the Dirac operator of the spectral triple $S T_{P S C}$ also has analytic continuation to a meromorphic function in the same region. Moreover, the assumption that the points $\{1,3\}$ are not poles of $\zeta_{\mathcal{L}}(s)$ ensures that $\zeta_{\mathcal{D}_{\mathcal{P}}}(s)$ has simple poles at these points. It also has a simple pole at $s=\sigma_{4}(\mathcal{P})$ by the last assumption. Thus, the spectral triple is regular (only simple poles) and the non-negative dimension spectrum is (2.20).

Proposition 2.9. Let $\mathcal{P}$ be a packing for which the three assumptions listed above hold. Then the asymptotic expansion of the spectral action for the spectral triple $S T_{P S C}(\mathcal{P})$ is of the form

$$
\operatorname{Tr}\left(f\left(\mathcal{D}_{\mathcal{P}} / \Lambda\right)\right) \sim \Lambda^{3} \zeta_{\mathcal{L}}(3) f_{3}-\Lambda \frac{1}{4} \zeta_{\mathcal{L}}(1) f_{1}+\Lambda^{\sigma}\left(\zeta\left(\sigma-2, \frac{3}{2}\right)-\frac{1}{4} \zeta\left(\sigma, \frac{3}{2}\right)\right) \mathcal{R}_{\sigma} f_{\sigma}
$$

where $\sigma=\sigma_{4}(\mathcal{P})$ the packing constant, $\mathcal{R}_{\sigma}=\operatorname{Res}_{s=\sigma} \zeta_{\mathcal{L}}(s)$ the residue of the zeta function of the fractal string $\mathcal{L}=\mathcal{L}(\mathcal{P})$, and $f_{\beta}=\int_{0}^{\infty} v^{\beta-1} f(v) d v$, the momenta of the test function.

Proof. Under the three assumptions listed above on the zeta function $\zeta_{\mathcal{L}}(s)$, the residues at the points $s=1$ and $s=3$ of the dimension spectrum are given, respectively, by

$$
\begin{aligned}
& \operatorname{Res}_{s=1} \zeta_{\mathcal{D}_{\mathcal{P}}}(s)=\frac{-1}{2} \operatorname{Res}_{s=1} \zeta\left(s, \frac{3}{2}\right) \cdot \zeta_{\mathcal{L}}(s)=-\frac{1}{2} \zeta_{\mathcal{L}}(1) \\
& \operatorname{Res}_{s=3} \zeta_{\mathcal{D}_{\mathcal{P}}}(s)=2 \operatorname{Res}_{s=3} \zeta\left(s-2, \frac{3}{2}\right) \cdot \zeta_{\mathcal{L}}(s)=2 \zeta_{\mathcal{L}}(3) .
\end{aligned}
$$


Thus, the terms in the asymptotic expansion of the spectral action

$$
\operatorname{Tr}\left(f\left(\mathcal{D}_{\mathcal{P}} / \Lambda\right)\right) \sim \sum_{\beta \in \Sigma_{\mathrm{ST}_{P S C}^{+}}} f_{\beta} \Lambda^{\beta} f\left|\mathcal{D}_{\mathcal{P}}\right|^{-\beta},
$$

with $f\left|\mathcal{D}_{\mathcal{P}}\right|^{-\beta}$ the residues, as in (1.3) , are given by

$$
\begin{aligned}
\operatorname{Tr}\left(f\left(\mathcal{D}_{\mathcal{P}} / \Lambda\right)\right) & \sim \Lambda^{3} \zeta_{\mathcal{L}}(3) \int_{0}^{\infty} v^{2} f(v) d v \\
& -\Lambda \frac{1}{4} \zeta_{\mathcal{L}}(1) \int_{0}^{\infty} f(v) d v \\
& +\Lambda^{\sigma}\left(\zeta\left(\sigma-2, \frac{3}{2}\right)-\frac{1}{4} \zeta\left(\sigma, \frac{3}{2}\right)\right) \operatorname{Res}_{s=\sigma} \zeta_{\mathcal{L}}(s) \int_{0}^{\infty} v^{\sigma-1} f(v) d v
\end{aligned}
$$

where $\sigma=\sigma_{4}(\mathcal{P})$.

Remark 2.10. The general question of when the zeta function $\zeta_{\mathcal{L}_{D}}(s)$ of the length spectrum $\mathcal{L}_{D}=\left\{a_{n, k}\right\}$ of an Apollonian packing $\mathcal{P}_{D}$ of $(D-1)$-dimensional spheres satisfies the three properties listed in Definition 2.7 above will be considered elsewhere.

2.9. Zeta regularization. The expression (2.21) for the spectral action of the sphere packing should be regarded as a "zeta regularized" form of the divergent series

$$
\mathcal{S}_{\mathcal{P}}(\Lambda)=\sum_{k=0}^{\infty} \mathcal{S}_{S_{a_{k}}^{3}}(\Lambda)
$$

that adds the contributions coming from the spectral actions of the individual spheres in the packing. Indeed, since the spectral action of an individual sphere is of the form

$$
\mathcal{S}_{S_{a_{n, k}}^{3}}(\Lambda)=\Lambda^{3} a_{n, k}^{3} f_{3}-\frac{1}{4} \Lambda a_{n, k} f_{1}+O\left(\left(\Lambda a_{n, k}\right)^{-K}\right),
$$

and both points 1 and 3 are smaller than the exponent of convergence $\sigma_{4}(\mathcal{P})$ of the series $\sum_{n, k} a_{n, k}^{s}$, the series

$$
\Lambda^{3} f_{3} \sum_{n, k} a_{n, k}^{3}-\frac{1}{4} \Lambda f_{1} \sum_{n, k} a_{n, k}
$$

is divergent and requires a suitable regularization. The spectral action (2.21) can be interpreted as such a regularization. Notice also that the error term $O\left(\left(\Lambda a_{n, k}\right)^{-K}\right)$ is very small for a fixed radius $a_{n, k}$ and for sufficiently large $\Lambda$, but when the radii $a_{n, k}$ vary over the set $\mathcal{L}(\mathcal{P})$ of lengths of the packing $\mathcal{P}$ it becomes large for any given $\Lambda$, so that (2.22) cannot be extended directly to the whole packing. The term

$$
\Lambda^{3} f_{3} \zeta_{\mathcal{L}}(3)-\frac{1}{4} \Lambda f_{1} \zeta_{\mathcal{L}}(1)
$$

in (2.21) is just a classical form of zeta regularization of the series (2.23), with the divergent $\sum a_{n, k}^{3}$ replaced by $\zeta_{\mathcal{L}}(3)$ and the divergent $\sum a_{n, k}$ replaced by $\zeta_{\mathcal{L}}(1)$. The additional term in (2.21), which depends on the residue of $\zeta_{\mathcal{L}}(s)$ at $s=\sigma_{4}(\mathcal{P})$ detects the presence of a fractal structure in the geometry. We discuss these issues further in $₫ 3$ below. 
2.10. Packed Swiss Cheese Spacetime spectral action. In the previous subsection we computed the asymptotic expansion of the spectral action for an Apollonian packing of 3 -spheres, under some assumptions on the behavior of the associated zeta function. Here we consider an associated (Euclidean) spacetime model. This generalizes to the case of a packing of spheres the simpler case of a single sphere $S^{3}$, where the associated spacetime is just $\mathbb{R} \times S^{3}$, with the Euclidean time line $\mathbb{R}$ compactified to a circle $S^{1}$ of size $\beta$. We generalize the form of the spectral action of $S_{\beta}^{1} \times S_{a}^{3}$, by replacing the 3 -sphere $S_{a}^{3}$ with a packing $\mathcal{P}$ of 3 -spheres $S_{a_{n, k}}^{3}$, and using the results in the previous section.

Proposition 2.11. Let $\mathcal{P}$ be a packing of 3-spheres satisfying the three conditions of Definition 2.7. Consider the product geometry $S_{\beta}^{1} \times \mathcal{P}$ of $\mathcal{P}$ with a circle of size $\beta$. Then the spectral action has asymptotic expansion with leading terms of the form

$$
\mathcal{S}_{S_{\beta}^{1} \times \mathcal{P}}(\Lambda) \sim 2 \beta\left(\Lambda^{4} \zeta_{\mathcal{L}}(3) \mathfrak{h}_{3}-\Lambda^{2} \frac{1}{4} \zeta_{\mathcal{L}}(1) \mathfrak{h}_{1}+\Lambda^{\sigma+1}\left(\zeta\left(\sigma-2, \frac{3}{2}\right)-\frac{1}{4} \zeta\left(\sigma, \frac{3}{2}\right)\right) \mathcal{R}_{\sigma} \mathfrak{h}_{\sigma}\right)
$$

where $\sigma=\sigma_{4}(\mathcal{P})$ is the packing constant (2.5), $\mathcal{R}_{\sigma}$ is the residue of $\zeta_{\mathcal{L}}(s)$ at $s=\sigma$, and

$$
\begin{gathered}
\mathfrak{h}_{3}:=\pi \int_{0}^{\infty} h\left(\rho^{2}\right) \rho^{3} d \rho, \quad \mathfrak{h}_{1}:=2 \pi \int_{0}^{\infty} h\left(\rho^{2}\right) \rho d \rho . \\
\mathfrak{h}_{\sigma}=2 \int_{0}^{\infty} h\left(\rho^{2}\right) \rho^{\sigma} d \rho .
\end{gathered}
$$

As observed in Lemma 2 of [8], the spectral action for $S_{\beta}^{1} \times S_{a}^{3}$, with the Dirac operator

$$
D_{S_{\beta}^{1} \times S_{a}^{3}}=\left(\begin{array}{cc}
0 & D_{S_{a}^{3}} \otimes 1+i \otimes D_{S_{\beta}^{1}} \\
D_{S_{a}^{3}} \otimes 1-i \otimes D_{S_{\beta}^{1}} & 0
\end{array}\right)
$$

is of the form

$$
\operatorname{Tr}\left(h\left(D_{S_{\beta}^{1} \times S_{a}^{3}}^{2} / \Lambda\right)\right) \sim 2 \beta \Lambda \operatorname{Tr}\left(\kappa\left(D_{S_{a}^{3}}^{2} / \Lambda\right)\right),
$$

for a test function $h(x)$, and with the test function $\kappa$ on the right-hand-side satisfying $\kappa\left(x^{2}\right)=\int_{\mathbb{R}} h\left(x^{2}+y^{2}\right) d y$. It then follows that the asymptotic expansion of the spectral action on $S_{\beta}^{1} \times S_{a}^{3}$ is given by (see Theorem 3 of [8])

$$
\operatorname{Tr}\left(h\left(D_{S_{\beta}^{1} \times S_{a}^{3}}^{2} / \Lambda\right)\right) \sim 2 \beta\left(\Lambda^{4} a^{3} \mathfrak{h}_{3}-\frac{1}{4} \Lambda^{2} a \mathfrak{h}_{1}\right),
$$

with the notation of (2.26). We now consider a similar situation, with the product geometry $S_{\beta}^{1} \times S_{a}^{3}$ replaced by $S_{\beta}^{1} \times \mathcal{P}$, where $\mathcal{P}$ is a packing of 3 -spheres satisfying the conditions of Definition 2.7. The Dirac operator of the product geometry is again of the form

$$
D_{S_{\beta}^{1} \times \mathcal{P}}=\left(\begin{array}{cc}
0 & \mathcal{D}_{\mathcal{P}} \otimes 1+i \otimes D_{S_{\beta}^{1}} \\
\mathcal{D}_{\mathcal{P}} \otimes 1-i \otimes D_{S_{\beta}^{1}} & 0
\end{array}\right),
$$

where $\mathcal{D}_{\mathcal{P}}$ is the Dirac operator of the spectral triple $S T_{P S C}$ described in Definition 2.3 . The same argument as in Lemma 2 of [8] shows that, as in (2.28)

$$
\operatorname{Tr}\left(h\left(D_{S_{\beta}^{1} \times \mathcal{P}}^{2} / \Lambda\right)\right) \sim 2 \beta \Lambda \operatorname{Tr}\left(\kappa\left(\mathcal{D}_{\mathcal{P}}^{2} / \Lambda\right)\right),
$$


with the test functions $h$ and $\kappa$ as above. Using the result of Proposition 2.9 we then obtain, as above, the expression (2.25), with $\mathfrak{h}_{\sigma}$ given by

$$
\mathfrak{h}_{\sigma}=\int_{\mathbb{R}_{+} \times \mathbb{R}} x^{\sigma-1} h\left(x^{2}+y^{2}\right) d x d y=\int_{0}^{\infty} h\left(\rho^{2}\right) \rho^{\sigma} d \rho \int_{-\pi / 2}^{\pi / 2} \cos (\theta) d \theta=2 \int_{0}^{\infty} h\left(\rho^{2}\right) \rho^{\sigma} d \rho
$$

In cosmological models based on the spectral action (see [27], [28]), the parameter $\beta$ is an artifact introduced by the choice of a compactification of the Euclidean time coordinate along a circle of size $\beta$. As discussed in $\S 3.1$ of [28], the parameter $\beta$ can be interpreted as an inverse temperature and related to the temperature of the cosmological horizon.

\section{Fractality scale truncation}

A realistic model of fractal structures in cosmology will necessarily involve a choice of scale at which fractality is cut off: while the universe may involve a fractal structure at the scale of galaxy superclusters and clusters, it does not appear fractal at our scales, hence the self-similarity property is expected to break down at some level. In a gravity model based on the spectral action, which already naturally involves a dependence on an energy scale $\Lambda$, it is natural to assume that the scale at which fractality breaks down will be in some way dependent on $\Lambda$. In the construction of the spectral triple of the PSCC model, discussed in $\$ 2.6$ above, we obtained a spectral action functional as a suitable kind of "zeta regularization" of the divergent series

$$
\sum_{n=0}^{\infty} \sum_{k=1}^{N_{n}} \mathcal{S}_{S_{a_{n, k}}^{3}}(\Lambda)
$$

where the sum is over all the 3 -spheres in the packing $\mathcal{P}$, with $a_{n, k}$ their radii, and with $N_{n}=6 \cdot 5^{n-1}$, the number of spheres in the $n$-th level of the packing construction. Indeed, as we have seen in the previous section, the spectral action $\mathcal{S}_{\mathcal{P}}(\Lambda)$ involves a zeta regularization of the above series, given by

$$
\left(\Lambda^{3} f_{3} \sum_{n, k} a_{n, k}^{3}-\frac{1}{4} \Lambda f_{1} \sum_{n, k} a_{n, k}\right)^{r e g}=\Lambda^{3} f_{3} \zeta_{\mathcal{L}}(3)-\frac{1}{4} \Lambda f_{1} \zeta_{\mathcal{L}}(1)
$$

and an additional term

$$
\Lambda^{\sigma}\left(\zeta\left(\sigma-2, \frac{3}{2}\right)-\frac{1}{4} \zeta\left(\sigma, \frac{3}{2}\right)\right) \mathcal{R}_{\sigma} f_{\sigma}
$$

involving the residue $\mathcal{R}_{\sigma}=\operatorname{Res}_{s=\sigma} \zeta_{\mathcal{L}}(s)$ at $\sigma=\sigma_{4}(\mathcal{P})$, which describes the fractality of the Apollonian packing.

3.1. Sphere counting function. In a model where fractality is truncated at a certain scale, one only considers the sphere packing $\mathcal{P}$ only up to a certain size. This requires estimating the number

$$
\mathcal{N}_{\alpha}(\mathcal{P})=\#\left\{S_{a_{n, k}}^{3} \in \mathcal{P}: a_{n, k} \geq \alpha\right\}
$$

of spheres in the given packing whose radii are of size at least $\alpha$. In the case of Apollonian packings of circles and of 2-spheres it is known, by a result of [5], that the Hausdorff dimension of the residual set of the packing is equal to

$$
\operatorname{dim}_{H}(\mathcal{R}(\mathcal{P}))=\lim _{\alpha \rightarrow 0}-\frac{\log \mathcal{N}_{\alpha}(\mathcal{P})}{\log \alpha}
$$


so that, for $\alpha \rightarrow 0$, one has $\mathcal{N}_{\alpha}(\mathcal{P}) \sim \alpha^{-\operatorname{dim}_{H}(\mathcal{R}(\mathcal{P}))+o(1)}$, see also [3]. It was proved in [21] that, in fact, one has the stronger $\mathcal{N}_{\alpha}(\mathcal{P}) \sim c_{\mathcal{P}} \alpha^{-\operatorname{dim}_{H}(\mathcal{R}(\mathcal{P}))}$. A general heuristic argument for the existence of a power law governing the behavior of the sphere counting function for sphere packings in arbitrary dimension is given in [1]. Let $\delta(\mathcal{P})$ denote the exponent of the power law, so that, for $\alpha \rightarrow 0$

$$
\mathcal{N}_{\alpha}(\mathcal{P}) \sim \alpha^{-\delta(\mathcal{P})+o(1)} .
$$

In fact, the result of [5] shows, in the case of an Apollonian packing of circles, that $\delta(\mathcal{P})$ is equal to the packing constant $\sigma_{2}(\mathcal{P})$, which combined with the result of [4] then gives the identification with the Hausdorff dimension. The general argument of $\S 2$ of $[5$ is independent of the dimension an it shows that, in general, one has the estimate

$$
\limsup _{\alpha \rightarrow 0}-\frac{\log \mathcal{N}_{\alpha}\left(\mathcal{P}_{D}\right)}{\log \alpha}=\sigma_{D}(\mathcal{P}) .
$$

Thus, if the sequence has a limit, then the limit has to be the packing constant $\sigma_{D}(\mathcal{P})$.

3.2. Spectral triple with truncation of fractality scale. Thus, in a cosmological model where fractality is truncated at a certain size $\alpha$, one would consider a spectral triple of the form

$$
\left(\mathcal{A}_{\mathcal{P}_{\alpha}}, \bigoplus_{n, k: a_{n, k} \geq \alpha} \mathcal{H}_{S_{a_{n, k}}^{3}}, \bigoplus_{n, k: a_{n, k} \geq \alpha} D_{S_{a_{n, k}}^{3}}\right),
$$

where $\mathcal{P}_{\alpha} \subset \mathcal{P}$ is the part of the packing that includes only those spheres $S_{a_{n, k}}^{3}$ with $a_{n, k} \geq \alpha$, and $\mathcal{A}_{\mathcal{P}_{\alpha}} \subset C\left(\mathcal{P}_{\alpha}\right)$ that satisfies the bounded commutator condition with the Dirac operator. Correspondingly, in this case, which involves only finitely many spheres, the spectral action would be of the form

$$
\mathcal{S}_{\mathcal{P}_{\alpha}}(\Lambda)=\sum_{n, k: a_{n, k} \geq \alpha} \mathcal{S}_{S_{a_{n, k}}^{3}}(\Lambda) .
$$

Lemma 3.1. Let $\mathcal{P}$ be a packing of 3-spheres satisfying the properties of Definition 2.7, and with the property that the function $F(\alpha)=-\log \left(\mathcal{N}_{\alpha}(\mathcal{P})\right) / \log (\alpha)$ has a limit for $\alpha \rightarrow 0$. Then the spectral action $\mathcal{S}_{\mathcal{P}_{\alpha}}(\Lambda)$ diverges at least like $\alpha^{-\left(\sigma_{4}(\mathcal{P})-1\right)+o(1)}$, when $\alpha \rightarrow 0$.

Proof. Each sphere contributes to the spectral action a term of the form

$$
\Lambda^{3} f_{3} \sum_{n, k} a_{n, k}^{3}-\frac{1}{4} \Lambda f_{1} \sum_{n, k} a_{n, k}+O\left(\left(\Lambda a_{n, k}\right)^{-K}\right) .
$$

Using the power law (3.2) for $\alpha \rightarrow 0$ we estimate

$$
\sum_{n, k} a_{n, k}^{3} \geq \alpha^{3-\delta(\mathcal{P})+o(1)} \quad \text { and } \quad \sum_{n, k} a_{n, k} \geq \alpha^{1-\delta(\mathcal{P})+o(1)} .
$$

By (3.3) and the hypothesis on the existence of the limit, we know that $\delta(\mathcal{P})=\sigma_{4}(\mathcal{P})$, which we know satisfies $\sigma_{4}(\mathcal{P})>3$, so that the exponents above are negative. Thus, for a fixed $\Lambda$ and for $\alpha \rightarrow 0$, the sum

$$
\sum_{n, k: a_{n, k} \geq \alpha}\left(\Lambda^{3} f_{3} \sum_{n, k} a_{n, k}^{3}-\frac{1}{4} \Lambda f_{1} \sum_{n, k} a_{n, k}\right)
$$

diverges at least like the dominant term $\alpha^{-(\delta(\mathcal{P})-1)+o(1)}$. 
3.3. Truncation estimates on the spectral action. One then expects that there will be a good approximation to the spectral action $\mathcal{S}_{\mathcal{P}_{\alpha}}(\Lambda)$ of the PSCC obtained by truncating fractality at a certain $\Lambda$-dependent scale. We discuss here possible choices of a function $\alpha=\alpha(\Lambda)$ that retain the property of having a good control on the error term of the spectral action $\mathcal{S}_{\mathcal{P}_{\alpha(\Lambda)}}(\Lambda)$. We first recall how one obtains explicit estimates for the error term in (2.17) of the spectral action on a 3-sphere, for a particular class of test functions, following the argument of Corollary 4 of [8].

Lemma 3.2. In the case of a cutoff function of the form $f(x)=P\left(\pi x^{2}\right) e^{-\pi x^{2}}$, where $P$ is a polynomial of degree $d$, the error term $\epsilon(\Lambda a)$ in the spectral action

$$
\mathcal{S}_{S_{a}^{3}}(\Lambda)=(\Lambda a)^{3} \int_{\mathbb{R}} v^{2} f(v) d v-\frac{1}{4}(\Lambda a) \int_{\mathbb{R}} f(v) d v+\epsilon(\Lambda a)
$$

satisfies the estimate

$$
|\epsilon(\Lambda a)| \leq(\Lambda a)^{3}\left(5+7 d+d^{2}\right) C_{Q} e^{-\frac{\pi}{2}(\Lambda a)^{2}}
$$

whenever $\Lambda a \geq \sqrt{(d+1)(1+\log (d+1))}$ and $\Lambda a \geq 1$. The coefficient $C_{Q}$ to be the sum of the absolute values of the coefficients of the polynomial $Q$, with $\hat{f}(x)=Q\left(\pi x^{2}\right) e^{-\pi x^{2}}$.

Proof. As in Corollary 4 of [8], we have $\hat{f}^{(2)}(x)=\left(x^{2} Z_{1}\left(\pi x^{2}\right)+Z_{2}\left(\pi x^{2}\right)\right) e^{-\pi x^{2}}$, where $Z_{1}=$ $-Q+2 Q^{\prime}-Q^{\prime \prime}$, and $Z_{2}=\frac{1}{2 \pi}\left(Q-Q^{\prime}\right)$, while generally $x^{k} e^{-x / 2} \leq 1$, for $x \geq 3 k(1+\log k)$. For $n \neq 0$ one then has

$$
\hat{f}(n \Lambda a)=Q\left(\pi(n \Lambda a)^{2}\right) e^{-\frac{\pi}{2}(n \Lambda a)^{2}} \cdot e^{-\frac{\pi}{2}(n \Lambda a)^{2}} \leq C_{Q} e^{-\frac{\pi}{2}(n \Lambda a)^{2}},
$$

because, by hypothesis, $\pi(n \Lambda a)^{2} \geq 3 d(1+\log d)$. Since the decay is more rapid than simply exponential, we can see that

$$
\begin{aligned}
2 \sum_{n=1}^{\infty} e^{-\frac{\pi}{2}(n \Lambda a)^{2}} & \leq 2 e^{-\frac{\pi}{2}(\Lambda a)^{2}}+2 \sum_{n=4}^{\infty} e^{-\frac{\pi}{2} n(\Lambda a)^{2}} \leq 2 e^{-\frac{\pi}{2}(\Lambda a)^{2}}+2 \frac{e^{-\frac{3 \pi}{2}(\Lambda a)^{2}}}{-1+e^{\frac{\pi}{2}(\Lambda a)^{2}}} \\
& \leq 2 e^{-\frac{\pi}{2}(\Lambda a)^{2}}+2 \frac{e^{-\frac{\pi}{2}(\Lambda a)^{2}} / 23}{-1+4.8} \leq 2.023 e^{-\frac{\pi}{2}(\Lambda a)^{2}}
\end{aligned}
$$

where we used the assumption that $\Lambda a \geq 1$. Thus, we have

$$
\sum_{n \neq 0} \hat{f}(n \Lambda a) \leq 2.023 C_{Q} e^{-\frac{\pi}{2}(\Lambda a)^{2}}
$$

Similarly, for $\hat{f}^{(2)}$ we get

$$
\sum_{n \neq 0} \hat{f}^{(2)}(n \Lambda a) \leq 2.023\left(2+3 d+\frac{1}{\pi} d^{2}\right) C_{Q} e^{-\frac{\pi}{2}(\Lambda a)^{2}} .
$$

By looking at the series for the spectral action after applying the Poisson summation formula, we see that the above terms contribute to the error as

$$
|\epsilon(\Lambda a)| \leq(\Lambda a)^{3} 2.023\left(2+3 d+\frac{1}{\pi} d^{2}\right) C_{Q} e^{-\frac{\pi}{2}(\Lambda a)^{2}}+\frac{2.023}{4} \Lambda a C_{Q} e^{-\frac{\pi}{2}(\Lambda a)^{2}},
$$

which can then be estimated from above as in (3.6).

We can then adapt the error estimates of Lemma 3.2 to the PSCC model, by performing a truncation on the fractality scale, dependent on the energy scale $\Lambda$. 
Remark 3.3. Under the assumption that $a_{n, k} \geq \alpha$, the error term in (3.5) is at most $O\left((\Lambda \alpha)^{-K}\right)$. Thus, it is natural to consider a model where the cutoff of fractality should happen at a scale $\alpha$ related to $\Lambda$ by the property that $\alpha(\Lambda) \cdot \Lambda$ grows like a positive power of $\Lambda$, so that one maintains a good control on the error term for large $\Lambda$.

Proposition 3.4. Consider a truncated packing $\mathcal{P}_{\alpha}$ of 3-spheres $S_{a_{n, k}}^{3}$ with $a_{n, k} \geq \alpha$, where $\alpha=\alpha(\Lambda)=\Lambda^{-1+\gamma}$ for some $0<\gamma<1$. Then the spectral action, computed using a test function of the form $f(x)=P\left(\pi x^{2}\right) e^{-\pi x^{2}}$ with $P$ a polynomial of degree $d$, satisfies

$$
\mathcal{S}_{\mathcal{P}_{\Lambda^{-1}}+\gamma}(\Lambda)=\left(\sum_{a_{n, k} \geq \Lambda^{-1+\gamma}} a_{n, k}^{3}\right) \Lambda^{3} f_{3}-\frac{1}{4}\left(\sum_{a_{n, k} \geq \Lambda^{-1+\gamma}} a_{n, k}\right) \Lambda f_{1}+\epsilon(\Lambda)
$$

where the error term satisfies

$$
|\epsilon(\Lambda)| \leq \Lambda^{3+\sigma(1-\gamma)} a_{\max }^{3}\left(5+7 d+d^{2}\right) C_{Q} e^{-\frac{\pi}{2} \Lambda^{2 \gamma}},
$$

where $a_{\max }=\max \left\{a_{n, k}\right\}$ is the largest radius in the packing $\mathcal{P}$, and $\sigma$ is the packing constant.

Proof. The estimate follows immediately from the previous Lemma, since we have

$$
\begin{gathered}
|\epsilon(\Lambda)| \leq \sum_{a_{n, k} \geq \Lambda^{-1+\gamma}} \Lambda^{3} a_{n, k}^{3}\left(5+7 d+d^{2}\right) C_{Q} e^{-\frac{\pi}{2}\left(\Lambda a_{n, k}\right)^{2}} \\
\leq \mathcal{N}_{\Lambda^{-1+\gamma}}(\mathcal{P}) \Lambda^{3} a_{\max }^{3}\left(5+7 d+d^{2}\right) C_{Q} e^{-\frac{\pi}{2} \Lambda^{2 \gamma}}=\Lambda^{3+\sigma(1-\gamma)} a_{\max }^{3}\left(5+7 d+d^{2}\right) C_{Q} e^{-\frac{\pi}{2} \Lambda^{2 \gamma}} .
\end{gathered}
$$

We consider a further possible way of truncating the spectral action, for spheres with smaller radii and the behavior of the error term. We start with the following error term estimate, for a single sphere.

Lemma 3.5. For a test function of the form $f(x)=P\left(\pi x^{2}\right) e^{-\pi x^{2}}$, for some polynomial $P$

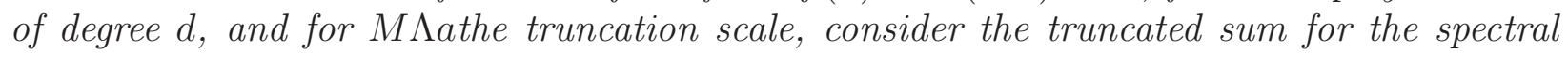
action,

$$
\mathcal{S}_{S_{a}^{3}, M}(\Lambda)=\sum_{\lambda<M \Lambda a} \operatorname{Mult}(\lambda) f\left(\frac{\lambda}{\Lambda a}\right)=\sum_{\lambda<M \Lambda a}\left(\lambda^{2}-\frac{1}{4}\right) f\left(\frac{\lambda}{\Lambda a}\right) .
$$

Let $N=\inf \left\{n \in \frac{1}{2}+\mathbb{Z} \mid n \geq \max \left\{M \Lambda a, \frac{3}{2}\right\}\right\}$. Then, assuming that

$$
\Lambda a \geq \sqrt{(d+1)(1+\log (d+1))} \quad \text { and } \quad \Lambda a \geq 1,
$$

the error term satisfies

$$
|\epsilon(\Lambda a)|=2 \sum_{\lambda \geq N}\left(\lambda^{2}-\frac{1}{4}\right) f\left(\frac{\lambda}{\Lambda a}\right) \leq \frac{2}{\pi}(\Lambda a)^{2}\left(1+\frac{2}{\pi}(\Lambda a)^{2}\right) C_{P} e^{-\frac{\pi}{2} M^{2}} .
$$

Proof. Since $u^{2}-\frac{1}{4}$ is a strictly positive quantity when evaluated at the integers, its absolute value is always less than $u^{2}$. Furthermore, all terms in the series are positive, so that we can work with $u^{2}$ instead of $u^{2}-\frac{1}{4}$ and our conclusions will remain valid. As in Lemma 3.2. we use the fact that $x^{k} e^{-\frac{x}{2}} \leq 1$, for all $x \geq 3 k(1+\log k)$. Then, as long as $x \geq$ $\sqrt{(d+1)(1+\log (d+1))}$, we know that $x^{2} f(x) \leq \frac{1}{\pi} C_{P} e^{-\frac{\pi}{2} x^{2}}$. 
Let our point of truncation be $M \Lambda a$, so that our sum is over $u<M \Lambda a$. Let $N$ be as in the statement. Then we have

$$
\begin{gathered}
|\epsilon(\Lambda a)|=2 \sum_{\lambda \geq N}\left(\lambda^{2}-\frac{1}{4}\right) f\left(\frac{\lambda}{\Lambda a}\right) \leq 2 \sum_{\lambda \geq N}(\Lambda a)^{2}\left(\frac{\lambda}{\Lambda a}\right)^{2} f\left(\frac{\lambda}{\Lambda a}\right) \\
\leq 2 \sum_{\lambda \geq N}(\Lambda a)^{2} \frac{1}{\pi} C_{P} e^{-\frac{\pi}{2}\left(\frac{\lambda}{\Lambda a}\right)^{2}} \leq \frac{2}{\pi}(\Lambda a)^{2} C_{P} \sum_{v=0}^{\infty} e^{-\frac{\pi}{2} \frac{1}{(\Lambda a)^{2}}\left(N^{2}+v\right)} \\
=\frac{2}{\pi}(\Lambda a)^{2} C_{P} e^{-\frac{\pi}{2}\left(\frac{N}{\Lambda a}\right)^{2}} \cdot \frac{1}{1-e^{-\frac{\pi}{2} \frac{1}{(\Lambda a)^{2}}}} \leq \frac{2}{\pi}(\Lambda a)^{2} C_{P} e^{-\frac{\pi}{2} M^{2}} \cdot \frac{1}{1-e^{-\frac{\pi}{2} \frac{1}{(\Lambda a)^{2}}}} .
\end{gathered}
$$

Now we check that $\left(1-e^{-\frac{\pi}{2} \frac{1}{(\Lambda a)^{2}}}\right)^{-1}<1+\frac{2}{\pi}(\Lambda a)^{2}$. Define $g(x)=\frac{1}{1-e^{-1 / x}}$. In the case $x \geq 1$, we have

$$
\begin{gathered}
e^{-1 / x}=\sum_{n=0}^{\infty} \frac{(-1)^{n} x^{-n}}{n !}=1-x^{-1}+\frac{1}{2} x^{-2}-\sum_{n=2}^{\infty} \frac{x^{1-2 n}}{(2 n-1) !}-\frac{x^{-2 n}}{(2 n) !} \\
=1-x^{-1}+\frac{1}{2} x^{-2}-\frac{x^{-2 n}}{(2 n-1) !} \sum_{n=2}^{\infty} x-\frac{1}{2 n} \leq 1-x^{-1}+\frac{1}{2} x^{-2}
\end{gathered}
$$

since every term in the third sum is positive. In the case $0<x<1$, substituting $u=1 / x$, with $u>1$, we have

$$
g(x)=\frac{1}{1-e^{-u}}=\frac{1}{1-e^{-u}} \frac{1+2 e^{-u}}{1+2 e^{-u}}=\frac{1+2 e^{-u}}{1+\left(e^{-u}-2 e^{-2 u}\right)} \leq 1+2 e^{-u} .
$$

Compare this with $1+1 / u$ : the term $2 e^{-u}$ decreases faster than $1 / u$, and $2 e^{-1}<1$, so for all $u>1$, we have $1+2 e^{-u}<1 / u$. Thus, for all $0<x<1$ we have $g(x) \leq 1+x$. Together, these two cases give the desired inequality

$$
\left(1-e^{\left.-\frac{\pi}{2} \frac{1}{(\Lambda a)^{2}}\right)^{-1}}<1+\frac{2}{\pi}(\Lambda a)^{2}\right.
$$

which then gives the stated estimate for the error term $|\epsilon(\Lambda a)|$.

For a given scale $M$, we consider a summation as above, of the form

$$
\sum_{\frac{3}{2 M}<\Lambda a_{n, k}<M} \sum_{\lambda=\frac{3}{2}}^{\left\lceil M \Lambda a_{n, k}\right\rceil-1} 2\left(\lambda^{2}-\frac{1}{4}\right) f\left(\frac{\lambda}{\Lambda a_{k}}\right) .
$$

We are interested now in the case of smaller spheres, with $a_{n, k}<\alpha$, where $\alpha=\alpha(\Lambda)$ was a previously chosen cutoff. Thus, we relate the scale $M$ to $\Lambda$ accordingly, by assuming that $M=M(\Lambda)=\alpha(\Lambda) \Lambda=\Lambda^{\gamma}$. This, in turn, gives the lower bound $\frac{3}{2 M}=\frac{3}{2} \Lambda^{-\gamma}$, which means that we are considering spheres with $a_{n, k} \geq \tilde{\alpha}(\Lambda)=\frac{3}{2} \Lambda^{-(1+\gamma)}$.

Proposition 3.6. For $\alpha(\Lambda)=\Lambda^{-1+\gamma}$ and $\tilde{\alpha}(\Lambda)=\frac{3}{2} \Lambda^{-(1+\gamma)}$, and for sufficiently large $\Lambda$, we have

$$
\mathcal{S}_{\mathcal{P}_{\tilde{\alpha}(\Lambda)}}(\Lambda)=\sum_{\frac{3}{2} \Lambda^{-\gamma}<\Lambda a_{n, k}<\Lambda^{\gamma}} \sum_{\lambda=\frac{3}{2}}^{\left\lceil\Lambda^{1+\gamma} a_{n, k}\right\rceil-1} 2\left(\lambda^{2}-\frac{1}{4}\right) f\left(\frac{\lambda}{\Lambda a_{k}}\right)+\epsilon(\Lambda)
$$


where

$$
|\epsilon(\Lambda)| \leq\left(\frac{3}{2}\right)^{\sigma} \frac{2}{\pi} C_{P} \Lambda^{2 \gamma+(1+\gamma) \sigma} e^{-\frac{\pi}{2} \Lambda^{2 \gamma}} .
$$

Proof. For large $\Lambda$ we can estimate the number $\mathcal{N}_{\tilde{\alpha}(\Lambda)}(\mathcal{P})$ of spheres with $a_{n, k} \geq \tilde{\alpha}(\Lambda)$, with $\tilde{\alpha}(\Lambda)^{-\sigma}=\left(\frac{3}{2}\right)^{\sigma} \Lambda^{(1+\gamma) \sigma}$, where $\sigma=\sigma_{4}(\mathcal{P})$ is the packing constant. For each sphere in the summation, we can apply the error term estimate of the previous lemma, with $\Lambda a_{n, k} \leq \Lambda^{\gamma}$ and we obtain

$$
|\epsilon(\Lambda)| \leq\left(\frac{3}{2}\right)^{\sigma} \Lambda^{(1+\gamma) \sigma} \frac{2}{\pi} C_{P} \Lambda^{2 \gamma}\left(1+\frac{2}{\pi} \Lambda^{2 \gamma}\right) e^{-\frac{\pi}{2} \Lambda^{2 \gamma}} .
$$

We can view the estimate of the error term of this sum as a way to estimate the effect of changing the cutoff scale from $\alpha(\Lambda)=\Lambda^{-1+\gamma}$ to $\tilde{\alpha}(\Lambda)=\frac{3}{2} \Lambda^{-(1+\gamma)}$.

\section{RELATED FRACTAL MODELS}

4.1. Fractal dodecahedra and cosmic topology. In [6], [27], 28, cosmological models based on the spectral action functional of gravity are constructed for (compactified, Euclidean) spacetimes of the form $S^{1} \times Y$ where $Y$ is either a spherical space form or a flat Bieberbach manifold, and it is shown that the spectral action detects the different cosmic topologies through the shape of an associated slow-roll inflation potential. In particular, it is shown in [6], [27], [38] that the spectral action for a spherical space form $Y=S^{3} / \Gamma$ is given by

$$
\mathcal{S}_{Y}(\Lambda)=\frac{1}{\# \Gamma} \mathcal{S}_{S^{3}}(\Lambda)
$$

independently of the spin structure (even though the Dirac spectrum itself changes for different spin structures). Of particular interest for cosmic topology is the case where $Y$ is the Poincaré homology 3-sphere (dodecahedral space), which is regarded as one of the most promising candidates for a non-simply connected cosmic topology, [12], 25].

We consider here a different possible model with fractal structure, where the building blocks are spherical dodecahedra, folded up to form Poincaré homology spheres, arranged in a fractal configuration that generalizes the Sierpinski fractal to dodecahedral geometry. Other similar construction can be done using other solids, and adapted to the other spherical space form candidates for cosmic topologies. These fractals are much simpler in structure than the Apollonian sphere packing described in the previous section, as the successive levels of the construction are all obtained by repeatedly applying the same uniform contraction factor. This makes the computation of the Hausdorff dimension immediate, as well as its identification with the exponent of convergence of the relevant zeta function. Moreover, it is also immediately clear that an analytic continuation exists to a meromorphic function on the entire complex plane, hence all the properties can be checked more easily.

More precisely, let $Y_{a}=S_{a}^{3} / \mathcal{I}_{120}$ be the quotient of a round 3-sphere of radius $a$ by the isometric action of the icosahedral group $\mathcal{I}_{120}$. The choice of a fundamental domain, given by a spherical dodecahedron, and the action of the group $\mathcal{I}_{120}$ determine a tiling of $S_{a}^{3}$ consisting of 120 dodecahedra. The quotient 3-manifold $Y_{a}$ is a Poincaré homology sphere, of volume $\operatorname{Vol}\left(Y_{a}\right)=\operatorname{Vol}\left(S_{a}^{3} / \mathcal{I}_{120}\right)=\frac{\pi^{2}}{60} a^{3}$.

Consider now the following well known construction of a Sierpinski type fractal based on the dodecahedron. Starting with an initial (solid) regular dodecahedron, one replaces it with 
20 new regular dodecahedra, contained inside the volume bounded by the initial one, each placed in the corner corresponding to one of the 20 vertices of the original dodecahedron. Each of the new dodecahedra is a copy of the original one scaled by a factor of $(2+\phi)^{-1}$ where $\phi$ is the golden ratio. One keeps iterating this procedure on each of the dodecahedra in the new configuration. Let $\mathcal{P}_{Y, n}$ be the union of dodecahedra obtained at the $n$-th step of the construction, where we simultaneously perform the identification of faces, in each dodecahedron, according to the action of $\mathcal{I}_{120}$, so that each is folded up into a Poincaré homology 3-sphere. Let $\mathcal{P}_{Y}$ denote the resulting limit, which in the set theoretic sense is given by the intersection $\mathcal{P}_{Y}=\cap_{n \geq 1} \mathcal{P}_{Y, n}$. The following fact then follows immediately.

Lemma 4.1. The Hausdorff dimension of the resulting set is

$$
\operatorname{dim}_{H} \mathcal{P}_{Y}=\frac{\log (20)}{\log (2+\phi)}=2.3296 \ldots
$$

This is equal to the exponent of convergence of the series

$$
\zeta_{\mathcal{L}\left(\mathcal{P}_{Y}\right)}(s)=\sum_{n \geq 0} 20^{n}(2+\phi)^{-n s}
$$

which has analytic continuation to a meromorphic function on $\mathbb{C}$,

$$
\zeta_{\mathcal{L}\left(\mathcal{P}_{Y}\right)}(s)=\frac{1}{1-20(2+\phi)^{-s}}
$$

with simple poles at the points

$$
\frac{\log (20)}{\log (2+\phi)}+\frac{2 \pi i m}{\log (2+\phi)}, \quad \text { with } \quad m \in \mathbb{Z}
$$

We then construct a spectral triple for the configuration $\mathcal{P}_{Y}$ using the same procedure as in [10]. Let $\left(C\left(Y_{a}\right), \mathcal{H}_{Y_{a}}, D_{Y_{a}}\right)$ is the spectral triple of $Y_{a}$ with $\mathcal{H}_{Y_{a}}=L^{2}\left(Y_{a}, \mathbb{S}\right)$ the square integrable spinors and $D_{Y_{a}}$ the Dirac operator. See [27], 38] for a more detailed discussion of these data.

Proposition 4.2. The spectral triple

$$
\left(\mathcal{A}_{\mathcal{P}_{Y}}, \mathcal{H}_{\mathcal{P}_{Y}}, \mathcal{D}_{\mathcal{P}_{Y}}\right)=\left(\mathcal{A}_{\mathcal{P}_{Y}}, \oplus_{n} \mathcal{H}_{Y_{a_{n}}}, \oplus_{n} D_{Y_{a_{n}}}\right)
$$

with $\mathcal{A}_{\mathcal{P}_{Y}} \subset C\left(\mathcal{P}_{Y}\right)$ satisfying the bounded commutator condition, and with $a_{n}=a(2+\phi)^{-n}$, has zeta function

$$
\zeta_{\mathcal{D}_{\mathcal{P}_{Y}}}(s)=\frac{a^{s}}{120}\left(2 \zeta\left(s-2, \frac{3}{2}\right)-\frac{1}{2} \zeta\left(s, \frac{3}{2}\right)\right) \zeta_{\mathcal{L}\left(\mathcal{P}_{Y}\right)}(s) .
$$

The positive part of the dimension spectrum is $\Sigma^{+}=\{1, \sigma, 3\}$, with $\sigma=\operatorname{dim}_{H}\left(\mathcal{P}_{Y}\right)$, while the full dimension spectrum $\Sigma$ also contains the points (4.2) off the real line. The metric dimension of the spectral triple is 3 .

Proof. The spectrum of the Dirac operator on the Poincaré homology sphere $Y_{a}$, with the correct multiplicities, can be computed explicitly using the method of [2] of generating functions, see [38]. In the case of the trivial spin structure, it is shown in [38] that one can separate 
the spectrum into 60 arithmetic progressions $\left\{\frac{3}{2}+k+60 j\right\}$ with multiplicities interpolated by 60 explicit polynomials $P_{k}\left(\frac{3}{2}+k+60 j\right)=\operatorname{Mult}\left(\frac{3}{2}+k+60 j\right)$, which satisfy

$$
\sum_{k=0}^{59} P_{k}(u)=\frac{1}{2} u^{2}-\frac{1}{8}
$$

This implies that

$$
\mathcal{S}_{Y_{a}}(\Lambda)=\sum_{k=0}^{59} \sum_{j \in \mathbb{Z}} P_{k}\left(\frac{3}{2}+k+60 j\right) f\left(\frac{\frac{3}{2}+k+60 j}{\Lambda}\right)=\frac{1}{120} \mathcal{S}_{S_{a}^{3}}(\Lambda)
$$

and that

$$
\zeta_{D_{Y_{a}}}(s)=\sum_{k=0}^{59} \sum_{j \in \mathbb{Z}} P_{k}\left(\frac{3}{2}+k+60 j\right)\left|\frac{3}{2}+k+60 j\right|^{-s}=\frac{1}{120} \zeta_{D_{S_{a}^{3}}}(s),
$$

hence (4.3) then follows as in Proposition 2.6.

Using the same technique that we used in the previous construction, we can then compute the leading terms in the asymptotic expansion of the spectral action.

Proposition 4.3. Let $\mathcal{P}_{Y}$ be the fractal arrangement described above. The spectral action functional for the spectral triple of Proposition 4.2 satisfies

$$
\operatorname{Tr}\left(f\left(\mathcal{D}_{\mathcal{P}_{Y}} / \Lambda\right)\right) \sim(\Lambda a)^{3} \frac{\zeta_{\mathcal{L}\left(\mathcal{P}_{Y}\right)}(3)}{120} f_{3}-\Lambda a \frac{\zeta_{\mathcal{L}\left(\mathcal{P}_{Y}\right)}(1)}{120} f_{1}+(\Lambda a)^{\sigma} \frac{\zeta\left(\sigma-2, \frac{3}{2}\right)-\frac{1}{4} \zeta\left(\sigma, \frac{3}{2}\right)}{120 \log (2+\phi)} f_{\sigma}
$$

where $\sigma=\operatorname{dim}_{H}\left(\mathcal{P}_{Y}\right)$.

Proof. The argument is exactly as in Proposition 2.9. where we now have $2<\sigma<3$ and the residue $\mathcal{R}_{\sigma}$ is given by

$$
\mathcal{R}_{\sigma}=\operatorname{Res}_{s=\sigma} \zeta_{\mathcal{L}\left(\mathcal{P}_{Y}\right)}(s)=\frac{1}{\log (2+\phi)}
$$

Corollary 4.4. Consider a (Euclidean, compactified) spacetime model of the form $S_{\beta}^{1} \times \mathcal{P}_{Y}$, with $\mathcal{P}_{Y}$ the fractal arrangement as above, and with $\beta$ the size of the compactification. Then the spectral action satisfies

$\mathcal{S}_{S_{\beta}^{1} \times \mathcal{P}_{Y}}(\Lambda) \sim 2 \beta\left(\Lambda^{4} \frac{a^{3} \zeta_{\mathcal{L}\left(\mathcal{P}_{Y}\right)}(3)}{120} \mathfrak{h}_{3}-\Lambda^{2} \frac{a \zeta_{\mathcal{L}\left(\mathcal{P}_{Y}\right)}(1)}{120} \mathfrak{h}_{1}+\Lambda^{\sigma+1} \frac{a^{\sigma}\left(\zeta\left(\sigma-2, \frac{3}{2}\right)-\frac{1}{4} \zeta\left(\sigma, \frac{3}{2}\right)\right)}{120 \log (2+\phi)} \mathfrak{h}_{\sigma}\right)$ with $\sigma=\operatorname{dim}_{H}\left(\mathcal{P}_{Y}\right)$ and $\mathfrak{h}_{1}, \mathfrak{h}_{3}, \mathfrak{h}_{\sigma}$ as in (2.26) and (2.27).

Proof. The result follows exactly as in Proposition 2.11. 


\section{Slow ROLL INFLATION IN FRACTAL UNIVERSES}

In the case of a compactified Euclidean spacetime of the form $S_{\beta}^{1} \times S_{a}^{3}$, it was observed in [8] that perturbations of the Dirac operator by a scalar field $D^{2} \mapsto D^{2}+\phi^{2}$ produce, in the calculation of the spectral action, a potential $V(\phi)$ for the scalar field, obtained as a combination of functions $\mathcal{V}\left(\phi^{2} / \Lambda^{2}\right)$ and $\mathcal{W}\left(\phi^{2} / \Lambda^{2}\right)$ with

$$
\begin{gathered}
\left.\operatorname{Tr}\left(h\left(\left(D^{2}+\phi^{2}\right) / \Lambda^{2}\right)\right)\right) \sim \pi \Lambda^{4} \beta a^{3} \int_{0}^{\infty} u h(u) d u-\frac{\pi}{2} \Lambda^{2} \beta a \int_{0}^{\infty} h(u) d u \\
+\pi \Lambda^{4} \beta a^{3} \mathcal{V}\left(\phi^{2} / \Lambda^{2}\right)+\frac{1}{2} \Lambda^{2} \beta a \mathcal{W}\left(\phi^{2} / \Lambda^{2}\right)
\end{gathered}
$$

where the functions are of the form

$$
\mathcal{V}(x)=\int_{0}^{\infty} u(h(u+x)-h(u)) d u, \quad \mathcal{W}(x)=\int_{0}^{x} h(u) d u
$$

It was observed in [27], [28] and [6], where the construction is generalized for spherical space forms and Bieberbach manifolds, that the resulting $V(\phi)$ has the form of a slow-roll inflation potential, and that the shape of the potential distinguishes between (almost all of) the different possible topologies and determined detectable signatures of the cosmic topology in the slow-roll parameters (which in turn determine spectral index and tensor-to-scalar ratio) and in the form of the power spectra for the scalar and tensor fluctuations. The result is similar in the case of the spherical space forms $Y_{a}=S_{a}^{3} / \Gamma$, with $V(\phi)$ replaced by $(\# \Gamma)^{-1} V(\phi)$, see [6], [27]. In this section we discuss how the inflation potential changed in the case of $S_{\beta}^{1} \times \mathcal{P}$, where $\mathcal{P}$ is either an Apollonian packing or 3-spheres or a configuration based on the fractal dodecahedron and the Poincaré homology sphere. In particular, we show that, in models of inflation based on the spectral action functional, the shape of the inflation potential changes depending on the fractal structure, hence the potential detect measurable effects of the presence and the type of fractal structure. In particular, it follows that, in a model of gravity based on the spectral action, the presence of fractality in the spacetime structure leaves a detectable signature in quantities, like the slow-roll parameters and the power spectra for the scalar and tensor fluctuations, that are in principle measurable in the $\mathrm{CMB}$, modulo the problem of determining the unknown parameter $\beta$ of the model, already discussed in [27], [28].

Proposition 5.1. Let $\mathcal{P}$ be either a packing of 3-spheres satisfying the three conditions of Definition 2.7, or a configuration of Poincaré homology 3-spheres arranged according to the fractal dodecahedron construction of \$4.1. Consider the product geometry $S_{\beta}^{1} \times \mathcal{P}$ of $\mathcal{P}$ with a circle of size $\beta$. Then the spectral action satisfies

$$
\begin{aligned}
\left.\operatorname{Tr}\left(h\left(\left(D^{2}+\phi^{2}\right) / \Lambda^{2}\right)\right)\right) \sim & 2 \beta\left(\Lambda^{4} \zeta_{\mathcal{L}}(3) \mathfrak{h}_{3}-\Lambda^{2} \frac{1}{4} \zeta_{\mathcal{L}}(1) \mathfrak{h}_{1}\right) \\
& +2 \beta \Lambda^{\sigma+1}\left(\zeta\left(\sigma-2, \frac{3}{2}\right)-\frac{1}{4} \zeta\left(\sigma, \frac{3}{2}\right)\right) \mathcal{R}_{\sigma} \mathfrak{h}_{\sigma} \\
+ & \pi \Lambda^{4} \beta \zeta_{\mathcal{L}}(3) \mathcal{V}\left(\phi^{2} / \Lambda^{2}\right)+\frac{\pi}{2} \Lambda^{2} \beta \zeta_{\mathcal{L}}(1) \mathcal{W}\left(\phi^{2} / \Lambda^{2}\right) \\
+ & 4 \beta \Lambda^{\sigma+1}\left(\zeta\left(\sigma-2, \frac{3}{2}\right)-\frac{1}{4} \zeta\left(\sigma, \frac{3}{2}\right)\right) \mathcal{R}_{\sigma} \mathcal{U}_{\sigma}\left(\phi^{2} / \Lambda^{2}\right),
\end{aligned}
$$

with

$$
\mathcal{V}(x)=\int_{0}^{\infty} u(h(u+x)-h(u)) d u, \quad \mathcal{W}(x)=\int_{0}^{x} h(u) d u
$$




$$
\mathcal{U}_{\sigma}(x)=\int_{0}^{\infty} u^{(\sigma-1) / 2}(h(u+x)-h(u)) d u,
$$

where $\sigma=\sigma_{4}(\mathcal{P})$ is the packing constant (2.5), $\mathcal{R}_{\sigma}$ is the residue of $\zeta_{\mathcal{L}}(s)$ at $s=\sigma$, and $\mathfrak{h}_{1}$, $\mathfrak{h}_{3}, \mathfrak{h}_{\sigma}$ are as in (2.26), (2.27).

Proof. The argument follows directly from Proposition 2.11, along the lines of Theorem 7 of 8]. We have

$$
\int_{0}^{\infty} h\left(\rho^{2}\right) \rho^{3} d \rho=\frac{1}{2} \int_{0}^{\infty} u h(u) d u,
$$

which gives rise to the term

$$
\int_{0}^{\infty} u(h(u+x)-h(u)) d u
$$

in the $\mathcal{V}$ part of the potential; similarly, we have

$$
\int_{0}^{\infty} h\left(\rho^{2}\right) \rho d \rho=\frac{1}{2} \int_{0}^{\infty} h(u) d u,
$$

which gives the term

$$
\int_{0}^{x} h(u) d u
$$

in the $\mathcal{W}$ part of the potential, and the term

$$
\int_{0}^{\infty} h\left(\rho^{2}\right) \rho^{\sigma} d \rho=\frac{1}{2} \int_{0}^{\infty} u^{\frac{\sigma-1}{2}} h(u) d u,
$$

which gives the term

$$
\int_{0}^{\infty} u^{\frac{\sigma-1}{2}}(h(u+x)-h(u)) d u
$$

in the $\mathcal{U}_{\sigma}$ part of the potential.

The slow-roll inflation potential $V(\phi)$ is obtained from the combination of functions $\mathcal{V}$, $\mathcal{W}, \mathcal{U}$ that appears in the expansion of the spectral action above.

Corollary 5.2. The function

$$
\begin{gathered}
\pi \Lambda^{4} \beta \zeta_{\mathcal{L}}(3) \mathcal{V}\left(\phi^{2} / \Lambda^{2}\right)+\frac{\pi}{2} \Lambda^{2} \beta \zeta_{\mathcal{L}}(1) \mathcal{W}\left(\phi^{2} / \Lambda^{2}\right) \\
+4 \beta \Lambda^{\sigma+1}\left(\zeta\left(\sigma-2, \frac{3}{2}\right)-\frac{1}{4} \zeta\left(\sigma, \frac{3}{2}\right)\right) \mathcal{R}_{\sigma} \mathcal{U}_{\sigma}\left(\phi^{2} / \Lambda^{2}\right)
\end{gathered}
$$

depends explicitly on the presence of fractality, through the coefficients $\zeta_{\mathcal{L}}(3), \zeta_{\mathcal{L}}(1)$, the residue $\mathcal{R}_{\sigma}$ and the packing constant $\sigma$.

\section{Conclusions}

In this paper we considered a model of gravity based on the spectral action functional. This is known to recover, via its asymptotic expansion, the usual Einstein-Hilbert action with cosmological term, along with modified gravity terms (conformal and Gauss-Bonnet gravity). We considered simple models of (Euclidean, compactified) spacetimes of the form $S_{\beta}^{1} \times \mathcal{P}$, where $\beta$ is the size of the $S^{1}$-compactification and $\mathcal{P}$ is a fractal configuration built out of 3 -spheres (Apollonian packings) or of other spherical space forms (Sierpinski fractals). We evaluated the leading terms of the asymptotic expansion of the spectral action, using 
information on the zeta function of the Dirac operator of a spectral triple, and we compared them, respectively, with the corresponding terms in the simpler case of $S_{\beta}^{1} \times S_{a}^{3}$ (spatial sections given by a single sphere of radius $a$ ) or $S_{\beta}^{1} \times Y$ where $Y$ is a spherical space form, in particular the Poincaré homology sphere (dodecahedral space). We regard the case of $S_{\beta}^{1} \times \mathcal{P}$, where $\mathcal{P}$ is an Apollonian packing of 3-spheres of a configuration obtained from a Sierpinski fractal dodecahedron, as a model of possible presence of fractality in spacetime geometry: a version of Packed Swiss Cheese Cosmology models. We showed that the resulting leading terms of the asymptotic expansion of the spectral action for $S_{\beta}^{1} \times \mathcal{P}$ differ from those of the ordinary $S_{\beta}^{1} \times S^{3}\left(\right.$ or $\left.S_{\beta}^{1} \times Y\right)$ case in two ways:

- The term $2 \Lambda^{4} \beta a^{3} \mathfrak{h}_{3}-\frac{1}{2} \Lambda^{2} \beta a \mathfrak{h}_{1}$, respectively corresponding to the cosmological and the Einstein-Hilbert term, are replaced by terms of the form $2 \Lambda^{4} \beta \zeta_{\mathcal{L}}(3) \mathfrak{h}_{3}-\frac{1}{2} \Lambda^{2} \beta \zeta_{\mathcal{L}}(1) \mathfrak{h}_{1}$, which can be seen as a zeta regularization of the divergent series of the 3 -sphere terms summed over the packing.

- There is an additional term in the gravity action functional of the form

$$
\Lambda^{\sigma+1}\left(\zeta\left(\sigma-2, \frac{3}{2}\right)-\frac{1}{4} \zeta\left(\sigma, \frac{3}{2}\right)\right) \mathcal{R}_{\sigma} \mathfrak{h}_{\sigma},
$$

where $\zeta(s, x)$ is the Hurwitz zeta function and $\sigma$ is the packing constant of $\mathcal{P}$, with $\mathcal{R}_{\sigma}$ the residue at $\sigma$ of the zeta function $\zeta_{\mathcal{L}}(s)$ of the fractal-packing. For a fractal dodecahedron $\sigma$ is the Hausdorff dimension, while for an Apollonian packing it is conjecturally the Hausdorff dimension of the residual set. In both cases this term detects modifications to the gravity action functional due to the presence of a fractal structure.

- The perturbation $D^{2} \mapsto D^{2}+\phi^{2}$ of the Dirac operator determines a slow-roll inflation potential $V(\phi)$ for the field $\phi$. The shape of the potential detects the presence of fractality through the coefficients $\zeta_{\mathcal{L}}(3), \zeta_{\mathcal{L}}(1)$, the packing constant $\sigma$, and the residue at $\sigma$ of $\zeta_{\mathcal{L}}(s)$.

Acknowledgment The first author was supported by a Summer Undergraduate Research Fellowship at Caltech. The second author is supported by NSF grants DMS-1007207, DMS1201512 , PHY-1205440.

\section{REFERENCES}

[1] T. Aste, Circle, sphere, and drop packing, Phys. Rev. E, Vol.53 (1996) N.3, 2571-2579.

[2] C. Bär, The Dirac operator on space forms of positive curvature, J. Math. Soc. Japan 48 (1996) N.1, 69-83.

[3] M. Borkovec, W. de Paris, R. Peikert, The fractal dimension of the Apollonian sphere packing, Fractals 2 (1994) N.4, 521-526.

[4] D. Boyd, The residual set dimension of the Apollonian packing, Mathematika 20 (1973) $170-174$.

[5] D. Boyd, The sequence of radii of the Apollonian packing, Math. Comp. 39 (1982) 249-254.

[6] B. Ćaćić, M. Marcolli, K. Teh, Coupling of gravity to matter, spectral action and cosmic topology, J. Noncommutative Geometry, Vol.8 (2014) N.2, 473-504.

[7] A. Chamseddine, A. Connes, The spectral action principle, Comm. Math. Phys. 186 (1997), no. 3, $731-750$.

[8] A. Chamseddine, A. Connes, The uncanny precision of the spectral action, Comm. Math. Phys. 293 (2010), no. 3, 867-897.

[9] A. Chamseddine, A. Connes, M. Marcolli, Gravity and the standard model with neutrino mixing, Advances in Theoretical and Mathematical Physics, 11 (2007) 991-1090. 
[10] E. Christensen, C. Ivan, M.L. Lapidus, Dirac operators and spectral triples for some fractal sets built on curves, Adv. Math. 217 (2008), N.1, 42-78.

[11] E. Christensen, C. Ivan, E. Schrohe, Spectral triples and the geometry of fractals, J. Noncommut. Geom. 6 (2012), no. 2, 249-274.

[12] S. Caillerie, M. Lachièze-Rey, J.P. Luminet, R. Lehoucq, A. Riazuelo, J. Weeks, A new analysis of the Poincaré dodecahedral space model, Astron. and Astrophys. 476 (2007) N.2, 691-696.

[13] A. Connes, Geometry from the spectral point of view. Lett. Math. Phys. 34 (1995), no. 3, 203-238.

[14] A. Connes, On the spectral characterization of manifolds, J. Noncommut. Geom. 7 (2013) N.1, 1-82.

[15] A. Connes, M. Marcolli, Noncommutative Geometry, Quantum Fields, and Motives, Colloquium Publications, Vol.55, American Mathematical Society, 2008.

[16] R.S. Farr, E. Griffiths, Estimate for the fractal dimension of the Apollonian gasket in d dimensions, Physical Review E 81 (2010) 061403 [4 pages]

[17] A. Gabrielli, F. Sylos Labini, M. Joyce, L. Pietronero, Statistical Physics for Cosmic Structures, Springer, 2005.

[18] R.L. Graham, J.C. Lagarias, C.L. Mallows, A.R. Wilks, C.H.Yan, Apollonian circle packings: number theory, J. Number Theory 100 (2003) 1-45.

[19] R.L. Graham, J.C. Lagarias, C.L. Mallows, A.R. Wilks, C.H.Yan, Apollonian Circle Packings: Geometry and Group Theory III. Higher Dimensions, Discrete Comput. Geom. 35 (2006) 37-72.

[20] J. Hawkes, Epsilon entropy and the packing of balls in Euclidean space, Mathematika 43 (1996) no. 1, $23-31$.

[21] A. Kontorovich, H. Oh, Apollonian circle packings and closed horospheres on hyperbolic 3-manifolds, Journal of AMS, Vol 24 (2011) 603-648.

[22] J.C. Lagarias, C.L. Mallows, A.R. Wilks, Beyond the Descartes circle theorem, Amer. Math. Monthly 109 (2002) 338-361.

[23] M.L. Lapidus, M. van Frankenhuijsen, Fractal geometry, complex dimensions and zeta functions. Geometry and spectra of fractal strings, Second edition. Springer Monographs in Mathematics. Springer, 2013.

[24] D.G. Larman, On the exponent of convergence of a packing of spheres, Mathematika 13 (1966) 57-59.

[25] J.P. Luminet, J. Weeks, A. Riazuelo, R. Lehoucq, Dodecahedral space topology as an explanation for weak wide-angle temperature correlations in the cosmic microwave background, Nature 425 (2003) 593-595.

[26] C. Mallows, Growing Apollonian packings, J. Integer Seq. 12 (2009) N.2, Article 09.2.1 [8 pages]

[27] M. Marcolli, E. Pierpaoli, K. Teh, The spectral action and cosmic topology, Comm. Math. Phys. 304 (2011), no. 1, 125-174

[28] M. Marcolli, E. Pierpaoli, K. Teh, The coupling of topology and inflation in noncommutative cosmology, Comm. Math. Phys. 309 (2012), no. 2, 341-369.

[29] H. Moraal, Apollonian arrangements of spheres in d-dimensional space, J. Phys. A 27 (1994) N.23, $7785-7791$.

[30] J.R. Mureika, C.C. Dyer, Multifractal analysis of Packed Swiss Cheese Cosmologies, General Relativity and Gravitation, Vol.36 (2004) N.1, 151-184.

[31] P. Olczykowski, A. Sitarz, On spectral action over Bieberbach manifolds, Acta Phys. Polon. B 42 (2011) N.6, 1189-1198.

[32] P.J.E. Peebles, Principles of Physical Cosmology, Princeton University Press, 1993.

[33] M.J. Rees, D.W. Sciama, Large-scale density inhomogeneities in the universe, Nature, Vol.217 (1968) $511-516$.

[34] M.B. Ribeiro, On Modeling a relativistic hierarchical (fractal) cosmology by Tolman's spacetime. I. Theory, The Astrophysical Journal, 388 (1992) 1-8.

[35] M.B. Ribeiro, On modeling a relativistic hierarchical (fractal) cosmology by Tolman's spacetime. II. Analysis of the Einstein-De Sitter model, The Astrophysical Journal, 395 (1992) 29-33.

[36] B. Söderberg, Apollonian tiling, the Lorentz group, and regular trees, Phys. Rev. A, Vol.46 (1992) N.4, $1859-1866$.

[37] F. Sylos Labini, M. Montuori, L. Pietroneo, Scale-invariance of galaxy clustering, Phys. Rep. Vol. 293 (1998) N. 2-4, 61-226.

[38] K. Teh, Nonperturbative spectral action of round coset spaces of SU(2), J. Noncommut. Geom. 7 (2013), no. $3,677-708$. 
Division of Physics, Mathematics and Astronomy, Caltech, 1200 E. California Blvd. Pasadena, CA 91125, USA

E-mail address: aaball@caltech.edu

E-mail address: matilde@caltech.edu 